$1-9-2015$

\title{
Asteroid (354) Eleonora: Plucking an odd duck
}

Michael J. Gaffey

University of North Dakota, gaffey@aero.und.edu

Vishnu Reddy

Sherry Fieber-Beyer

University of North Dakota, sherry.fieber.beyer@und.edu

Edward Cloutis

How does access to this work benefit you? Let us know!

Follow this and additional works at: https://commons.und.edu/ss-fac

Part of the Astrophysics and Astronomy Commons

\section{Recommended Citation}

Michael J. Gaffey, Vishnu Reddy, Sherry Fieber-Beyer, et al.. "Asteroid (354) Eleonora: Plucking an odd duck" (2015). Space Studies Faculty Publications. 2.

https://commons.und.edu/ss-fac/2

This Article is brought to you for free and open access by the Department of Space Studies at UND Scholarly Commons. It has been accepted for inclusion in Space Studies Faculty Publications by an authorized administrator of UND Scholarly Commons. For more information, please contact und.commons@library.und.edu. 


\title{
Asteroid (354) Eleonora: Plucking an odd duck
}

\author{
Michael J.Gaffey,VishnuReddy,SherryFieber-Beyer, EdwardCloutis
}

\begin{abstract}
During a survey of the S-type asteroids, Gaffey et al. (Gaffey, M.J., Bell, J.F., Brown, R.H., Burbine, T.H., Piatek, J., Reed, K.L., Chaky, D.A. [1993]. Icarus 106, 573-602) identified Asteroid (354) Eleonora as anomalous with a $1 \mu \mathrm{m}$ absorption feature $\sim 2.5$ times stronger than any S-asteroid of comparable size. Subsequent investigation revealed significant differences in the $1 \mu \mathrm{m}$ absorption feature between the visible \& very near-infrared CCD spectra $(\lambda<\sim 1.0 \mu \mathrm{m})$ and other spectral data sets for this asteroid. There were also significant spectral differences among the several CCD survey spectra (SMASS-I, SMASS-II \& $\mathrm{S}^{3} \mathrm{OS}^{2}$ ) of Eleonora. These differences could potentially arise from spectral variations across the asteroid surface, from observational phase angle differences, from surface temperature differences, from viewing geometry for a nonspherical body, or from the use of standard stars with deviated to different degrees from a true solar standard.
\end{abstract}

In June 2011 Asteroid (354) Eleonora was observed over two nights using the NASA Infrared Telescope Facility (IRTF) at Mauna Kea Observatory in order to test these possible scenarios and to better understand the nature and history of Eleonora and its relationships to other asteroids and to the meteorites. Analysis of this data set has eliminated the following options as the cause of the differences in the $1 \mu \mathrm{m}$ absorption feature within the CCD data sets and between the CCD data sets and the other spectral data: (1) rotational spectral variations; (2) variation in surface composition with latitude; (3) observation phase; (4) surface temperature variations with differing heliocentric distance in the asteroid's elliptical orbit; (5) spectral effects of viewing geometry for a nonspherical body; and (6) differences in spectral standard stars. We conclude that none of the CCD spectra of (354) Eleonora are reliable, and that within the limits of their spectral coverage, analyses of the three CCD spectra would produce significantly different - and generally unreliable - indications of surface mineralogy. An effort needs to be made to determine whether "bad" CCD spectra are rare with the case of (354) Eleonora being an uncommon occurrence or whether there is a broader problem with the CCD asteroid survey data sets, and if so, how to identify the "bad" spectra.

While CCD survey spectra show apparently irreconcilable differences, the near-infrared spectra of (354) Eleonora from various observers show only minor differences, primarily in the overall spectral slope, most of which can be attributed to slight differences in the standard stars used to calibrate the data.

In June 2011, 226 near-infrared $(\sim 0.76-2.5 \mu \mathrm{m})$ spectra of (354) Eleonora were obtained using the SpeX instrument on the NASA Infrared Telescope Facility at Mauna Kea Observatory. These spectra were consistent with the six sets of NIR spectra obtained for Eleonora by previous observers. The primary variation observed in this new data set was an approximately $10 \%$ variation in spectral slope between $\sim 0.8 \mu \mathrm{m}$ and $\sim 1.6 \mu \mathrm{m}$ during the rotation period of the asteroid.

Mineralogically diagnostic spectral parameters extracted from this new data are most consistent with a surface assemblage of fine-grained intimately mixed olivine $\left(\sim 60-70 \%, \sim\right.$ Fo $\left._{61-71}\right)$ and low nickel $(<\sim 7-$ $8 \% \mathrm{Ni}) \mathrm{NiFe}$ metal. The Fo estimate is consistent with previous estimates $\left(\mathrm{Fo}_{66 \pm 5}\right)$ by Sanchez et al. (Sanchez, J.A., Reddy, V., Kelley, M.S., Cloutis, E.A., Bottke, W.F., Nesvorný, D., Lucas, M.P., Hardersen, P.S., Gaffey, M.J., Abell, P.A., Le Corre, L. [2014]. Icarus 228, 288-300), but not with the estimate ( $\left.\sim \mathrm{Fog}_{90}\right)$ of Sunshine et al. (Sunshine, J.M., Bus, S.J., Corrigan, C.M., McCoy, T.J., Burbine, T.H. [2007]. Meteorit. Planet. Sci. 42, 155-170). The surface assemblage appears to contain a small 
component $(\sim 8-10 \%)$ of igneous pyroxene (weakly constrained at $\left.\sim \mathrm{Fs}_{50} \mathrm{Wo}_{10}\right)$. The parent lithology of the surface regolith may be similar to a pallasite assemblage, although none of the three known types of pallasites are good mineralogical matches.

\section{Keywords}

Asteroids, Spectroscopy, Mineralogy

\section{Introduction}

Asteroid (354) Eleonora is a moderately large object (diameter $=155 \mathrm{~km}$ ) located in the middle of the asteroid belt $(\mathrm{a}=2.796 \mathrm{AU}-\mathrm{JPL}$ Horizons Website). It has an eccentricity ( $\mathrm{e}=0.1157-\mathrm{JPL}$ Horizons Website) near the average for main belt asteroids. Its inclination ( $\mathrm{i}=18.4^{\circ}-\mathrm{JPL}$ Horizons Website) is higher than $\sim 85 \%$ of the 931 brightest $(\mathrm{H} \leqslant 10.8$ ) main belt asteroids (Minton and Malhotra, 2010). Spectral investigations have identified it as an olivine-rich object probably produced by igneous processes within its parent body.

Eleonora presents an interesting enigma. In most taxonomies based on shorter wavelength spectral data (e.g., $\lambda \sim 0.4$ to $<1.0 \mu \mathrm{m}$; ECAS, 24 -filter, CCD data) it has been regularly classified taxonomically as some variant of the S-type (Table 1). However, its $1 \mu \mathrm{m}$ absorption band intensity is $\sim 2.5$ times the intensity that would be expected for an S-asteroid of its size (Fig. 1 - Gaffey et al., 1993). Eleonora had the strongest $1 \mu \mathrm{m}$ absorption band among the forty S-asteroids in the 52-color survey (Bell et al., 1988). Note: It's not possible to get an accurate $1 \mu \mathrm{m}$ band depth (i.e. relative to the spectral continuum) from CCD spectra because the spectral continuum cannot be robustly determined without spectral coverage that extends to $\sim 1.5 \mu \mathrm{m}$ or at least beyond the long wavelength limit of the $1 \mu \mathrm{m}$ absorption band.

Table 1. Taxonomic classifications of (354) Eleonora.

\begin{tabular}{|l|l|l|}
\hline Reference & Spectral data & Taxonomic class \\
\hline Tholen (1984, 1989) & ECAS & S \\
\hline Barucci et al. (1987) & ECAS & S2 \\
\hline Tedesco et al. (1989) & UVx \& albedo & S \\
\hline Burbine (1991) & ECAS \& 52-color & S - Anom \\
\hline Howell et al. (1994) & ECAS \& 52-color & So \\
\hline Bus and Binzel (2002b) & CCD & Sl \\
\hline Lazzaro et al. (2004) & CCD & A/S \\
\hline DeMeo et al. (2009) & SpeX 0.8-2.5 $\mu \mathrm{m}$ & A \\
\hline
\end{tabular}

a. Lazzaro et al. (2004) classified (354) Eleonora as both an S-type using "Tholen" criteria and as an A-type using "Bus" criteria. Eleonora was classified as an S(I) object (olivine-dominated) in the mineralogy based system of Gaffey et al. (1993). 


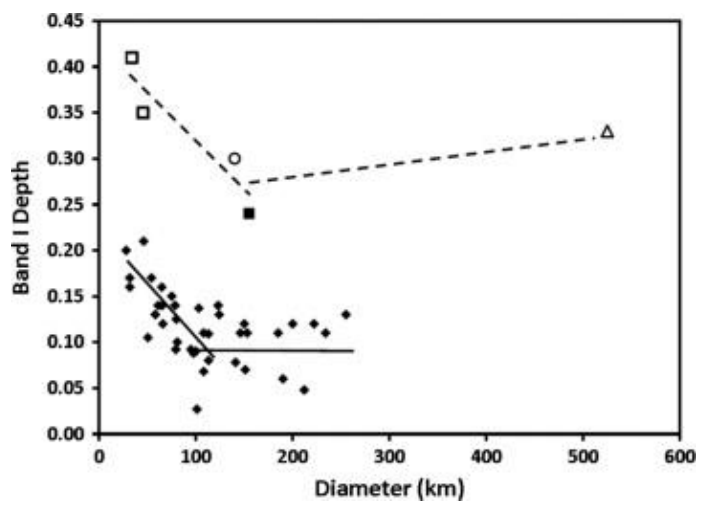

Fig. 1. Band depth versus diameter for S-asteroids (small solid diamonds, 354 Eleonora (large solid square), Vtype Asteroid (4) Vesta (open triangle), R-type Asteroid (349) Dembowska (open circle), and two A-type Asteroids (289 Neneta \& 446 Aeternitas - open squares). Lines indicate apparent trends. Modified from Gaffey et al. (1993).

Extended spectral coverage (e.g., $\lambda \sim 0.7-2.5 \mu \mathrm{m}$ ) clearly shows that Eleonora has an olivine dominated assemblage. Taxonomically that would make it an A-type or an $\mathrm{S}(\mathrm{I})$ subtype in the mineralogical classification of Gaffey et al. (1993). In the original Tholen taxonomy (Tholen, 1984, Tholen and Barucci, 1989) based on the Eight Color Asteroid Survey (ECAS) data (Zellner et al., 1985) and albedos, A-types corresponded to olivine-rich assemblages. Most subsequent taxonomies based on CCD data were forced to rely on less diagnostic criteria since that data generally lacked the longer wavelength coverage which allowed the ECAS based system to robustly distinguish between olivine assemblages and olivinepyroxene assemblages. If, as seems reasonable, A-type asteroids are distinguished from most S-type asteroids by the absence of a $\sim 2 \mu \mathrm{m}$ pyroxene feature, then A-types classified based of CCD data need to be treated with caution (e.g., 4142 Dersu-Uzala which is classified as an A-type based on CCD data (Bus and Binzel, 2002b) but which shows a well-defined $2 \mu \mathrm{m}$ pyroxene feature (Binzel et al., 2004)).

An obvious question is (a) whether there is a real difference between the predominantly olivine Sasteroids (i.e., subtype S(I) - Gaffey et al., 1993) and the predominantly olivine A-asteroids or (b) whether these two types are parts of a continuous trend. Except for (354) Eleonora, there is a distinct gap between the band depths of A- and S-type asteroids (Fig. 1), which would suggest that they do not form a continuous sequence from A- to S-type. This is not merely an issue of taxonomic interest but would reflect real geologic differences in the parent body history and the formation processes of the $S$-type and the A-type objects.

\section{Eleonora's diverse spectra}

The second enigmatic factor concerning Asteroid (354) Eleonora is the diverse set of reflectance spectra which have been obtained for this object by different observers. Spectra of Eleonora at short visible and very-near-infrared wavelengths $(\lambda<1 \mu \mathrm{m})$ have previously been obtained by five different groups of observers (Fig. 2). Spectra at near-infrared (NIR) wavelengths $(\lambda \sim 0.8-2.5 \mu \mathrm{m})$ have also been obtained by five groups of observers (Fig. 3). The short wavelength spectra (Fig. 2) show differences significant enough to result in very different mineralogical interpretations. 


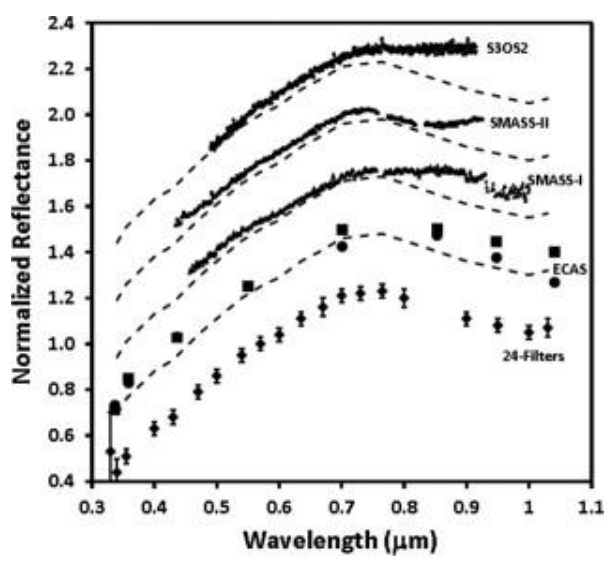

Fig. 2. Shorter wavelength spectra of (354) Eleonora. Spectra have been normalized to around $0.56 \mu \mathrm{m}$, and offset vertically by 0.2 for clarity. The dashed line is a curve corresponding to the 24 -filter data and is used for comparisons.

Sources: 24-filter data (Chapman and Gaffey, 1979); ECAS/Eight Color Asteroid Survey (Zellner et al., 1985); SMASS-I (Xu et al., 1995); SMASS-II (Bus and Binzel, 2002a); S3OS2 (Lazzaro et al., 2004). Digital versions of all data sets are available from the Small Bodies Node of The Planetary Data System (http://sbn.psi.edu).

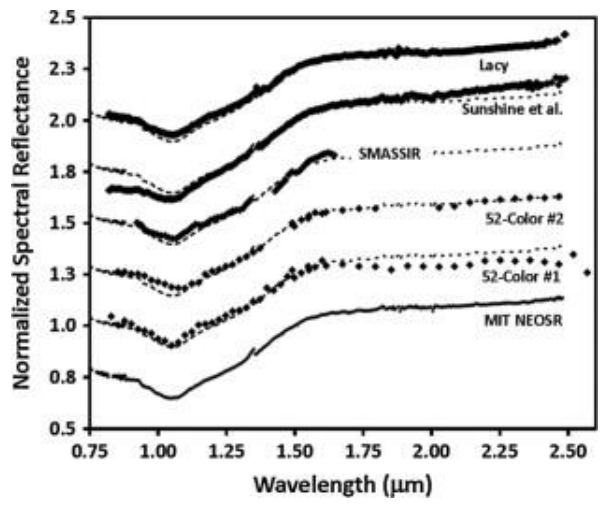

Fig. 3. Near-infrared reflectance spectra of (354) Eleonora obtained by five different groups. Spectra have been normalized to $1.5 \mu \mathrm{m}$ and offset 0.25 for clarity. The dashed line is the MIT NEORS spectrum used for comparison

Sources: MIT NEOSR (MIT-UH-IRTF Joint Campaign for NEO Spectral Reconnaissance); 52-color (Bell et al., 1988); SMASSIR (Burbine and Binzel, 2002), Sunshine et al. (2007), and Lacy (Gietzen et al., 2012). Digital data for the 52-color and Sunshine et al. are available from the PDS-SBN. The MIT NEOSR and SMASSIR data are available on the MIT Planetary Spectroscopy website (http://smass.mit.edu). The Lacy spectral data was provided by Claud Lacy of the University of Arkansas, Fayetteville.

The 24-filter data and the ECAS data (Fig. 2) are in general agreement with a reasonably strong (depth $\sim 0.1-0.2) \sim 1 \mu \mathrm{m}$ absorption feature longwards of $\sim 0.75 \mu \mathrm{m}$. None of the CCD data show a similar feature (Fig. 2). The S3OS2 spectrum is essentially flat beyond $\sim 0.7 \mu \mathrm{m}$. The SMASS-II spectrum shows a much weaker feature (depth $\sim 0.06$ ) with a minimum near $0.85 \mu \mathrm{m}$. The SMASS-I spectrum is essentially flat between $\sim 0.7$ and $0.92 \mu \mathrm{m}$, then drops stepwise to a depth of $\sim 0.08$. The position and even the presence of a $\sim 1 \mu \mathrm{m}$ absorption feature is ambiguous in the CCD data.

The near-infrared (NIR) spectra (Fig. 3) show more subtle variations. All the observations agree on the presence of $\mathrm{a} \sim 1 \mu \mathrm{m}$ absorption feature, although there are differences in the band depth. There are also differences in the spectral slope beyond $\sim 1.6 \mu \mathrm{m}$. It appears that the $\sim 2 \mu \mathrm{m}$ absorption feature is either absent or very weak. Compared to the short wavelength data sets, the NIR data is generally consistent, and the relatively subtle variations could be attributable to several potential sources of variations. The 
NIR spectra all indicate an assemblage dominated by olivine, which taxonomically would be classified as an A-type.

\section{Implications}

Any olivine-dominated spectrum indicates a very high physical abundance of olivine within the silicate assemblage, since the presence of even comparably small amounts of other silicate minerals such as pyroxene would substantially modify the resulting spectrum. The rare olivine-dominated R-type chondrites (Rumurutites - Kallemeyn et al., 1996) show a strong olivine feature in their spectra (e.g., Berlin, 2003, Berlin et al., 2003, Sunshine et al., 2007, Cloutis et al., 2012a). Finer particle size fractions of CK chondrites also exhibit a nearly pure olivine absorption feature albeit much weaker (Cloutis et al., 2012b). No other chondrite spectra show the strong olivine features of nearly pure olivine assemblages (Fig. 4). Generally, mono-minerallic olivine assemblages produced by igneous processes can exhibit such spectra. Among the differentiated meteorites, two types show predominately olivine spectra: brachinites and some of the angrites (Fig. 4). Such igneous assemblages are generally either: (i) olivine-rich partial melt residues (restites) or (ii) olivine cumulates formed by the settling of olivine crystals during the slow cooling of a mafic magma. The ureilites also represent olivine-rich residues of the partial melting of chondritic precursors (e.g., Goodrich et al., 2007) but are not spectrally dominated by olivine (Cloutis et al., 2012b). The compositions of the olivine and of accessory minerals - such as pyroxene or spinel often allow these different mechanisms to be distinguished from each other, and can potentially provide keys into the origin and history of the olivine-rich asteroid.

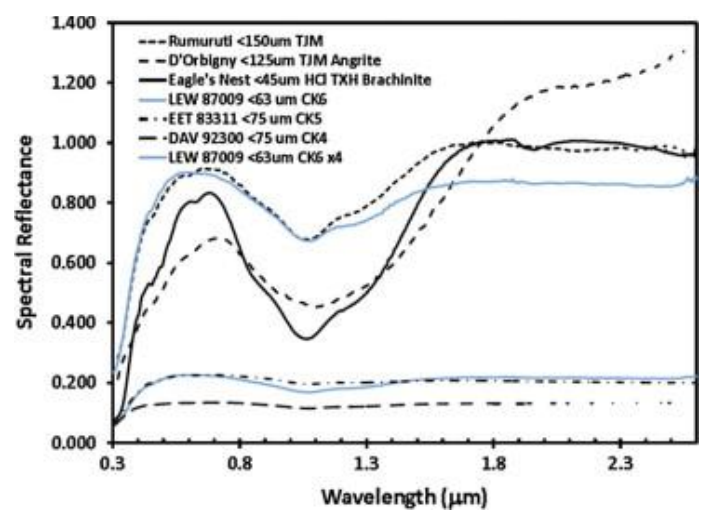

Fig. 4. Olivine-dominated reflectance spectra of four types of predominantly olivine meteorites. Actual reflectance (albedo) depends on particle size and the presence or absence of a strongly absorbing species. Digital spectral data are from the RELAB spectral database: Rumuruti (Sunshine et al., 2007; Sample MT JTM-013); D’Orbigny (Burbine et al., 2006; Sample TB-TJM-063); Eagle's Nest (Sample MT-TXH-56-A); LEW 87009 (Sample MP-LAM-005); EET 83311 (Sample PH-D2M-047); and DAV 92300 (Sample PHD2M-053). Since brachinites are all finds, most have experienced significant terrestrial weathering. As indicated in the RELAB database, Hiroi cleaned the Eagle's Nest sample with hydrochloric acid and ultrasound to remove iron oxide weathering products. The spectral curve of the CK6 chondrite LEW 87009 was multiplied by a factor of four to emphasize the characteristic olivine spectrum and is plotted as the upper blue curve. (For interpretation of the references to color in this figure legend, the reader is referred to the web version of this article.)

\section{Possible explanations for the spectral heterogeneity}

The diversity among the measured spectra of (354) Eleonora could arise from at least seven different causes. These are outlined below and five are tested in subsequent sections.

A. The surface of Eleonora could be longitudinally heterogeneous. Since most observations are snapshots, taken during a period much shorter than the rotational period of the asteroid (i.e., 
minutes versus hours), the resulting spectra are for essentially one face of the object. If the surface is spectrally heterogeneous then spectra taken at different rotational phases would be different. In that case, observations taken at similar rotational phases should be similar, and the spectral differences should be repeatable with rotational phase. Unfortunately this option cannot be tested for the previous spectral data because the rotational period of (354) Eleonora is not known with sufficient precision to establish the relative rotational phase for observations spaced years apart. The new observations discussed below will test this hypothesis.

B. The surface mineralogy of Eleonora could be latitudinally heterogeneous with spectral differences between the northern and southern hemispheres. In this model, the observed spectra would vary with the sub-Earth latitude (SEL) of the observations. In such a case, spectra obtained at high northern latitudes would differ from those taken at high southern latitudes and from those taken at equatorial aspects. In this scenario, the spectra would vary with SEL and be repeatable for similar SELs. This hypothesis will be tested using the available data sets.

C. The spectral difference could reflect observations at different phase angles. The phase angle (Sun-asteroid-observer angle) of individual observations will affect what portions of the asteroid are simultaneously illuminated and in view for the observer. Gradie and Veverka (1986) showed the effects on spectra of varying phase angle. Reddy et al. (2012) and Sanchez et al. (2012) have documented changes in spectral slopes and band depths for Asteroid (4) Vesta and several near-Earth asteroids with observational phase angle. This possibility can be tested for (354) Eleonora using the available data sets.

D. The difference could be related to temperature differences of the Eleonora surface between the various observations. Reflectance spectra of silicate assemblages in general and olivinedominated assemblages in particular vary with temperature (e.g., Roush, 1984, Roush and Singer, 1986, Lucey et al., 1998, Moroz et al., 2000, Hinrichs and Lucey, 2002, Sunshine et al., 2007, Reddy et al., 2011a, Reddy et al., 2011b). The eccentricity of Eleonora's orbit (0.1157) results in perihelion and aphelion distances of 2.473 and 3.120 AUs respectively. Using the equation for asteroid surface temperature from Burbine et al. (2009):

$\mathrm{T}=[(1-\mathrm{A}) \mathrm{Lo} / 16 \eta \varepsilon \sigma \pi \mathrm{r} 2] 1 / 4$

where $A$ is the asteroid albedo, $L o$ is the solar luminosity $\left(3.839 \times 10^{26} \mathrm{~W}\right), \eta$ is the beaming factor (assumed to be 1.0), $\varepsilon$ is the asteroid's infrared emissivity (assumed to be 0.9), $\sigma$ is the Stefan-Boltzmann constant, and $r$ is the asteroid's distance from the Sun in meters. For the measured albedo $(0.1948)$ and at opposition (phase angle $=0^{\circ}$ ), the effective perihelion and aphelion temperatures of Eleonora would be $172 \mathrm{~K}$ and $153 \mathrm{~K}$, respectively. The expected spectral variations in olivine-dominated spectra versus the Eleonora surface temperatures for these observations can be used to test this idea.

E. The variations could be shape related. The spectrum of an ellipsoid or otherwise non-spherical body will vary between the side view and the end-on view (Gradie and Veverka, 1981, Gradie and Veverka, 1982). Although it is not possible to establish the rotational phase of the spectral observations to the lightcurve - and hence to the viewing aspect - the magnitude of the effect can be tested. 
F. Spectral difference could potentially arise from the use of standard stars which differ from solar to different degrees. This effect can be tested from the new and existing data.

G. There could be some problems - systematic or otherwise - that produced spurious spectral deviations in some these CCD spectra. Elimination of the other options would focus attention on this possibility. In that case, it would be important - if possible - to identify the source of the problem and establish some means of identifying which spectra are not reliable and to what degree.

\section{Testing the hypotheses}

In particular, we are concerned with the substantial variations in the $\sim 1 \mu \mathrm{m}$ absorption feature seen in the short wavelength spectra, and we will focus our attention on identifying the cause of these spectral differences. The smaller variations in the NIR spectra will be addressed as appropriate.

\subsection{Testing for longitudinal compositional heterogeneity}

Except for pole-on viewing geometries, rotation of an asteroid sequentially exposes increasing longitudes to the observer. During one rotation period, the sub-Earth (sub-observer) longitude exposes the full longitude range to the observer. At any given rotational phase and at zero phase angle, one half of the object is both illuminated and in view. With increasing phase angle, less of the illuminated hemisphere is in view. For example, at $90^{\circ}$ phase (quarter Moon) only half of the lunar disk is illuminated. Although near-Earth asteroids can be observed at very high phase angles (e.g., $>90^{\circ}$ ), the maximum phase angle for observations of main belt asteroids is generally less than $30^{\circ}$. For (354) Eleonora, the maximum phase angle is $24^{\circ}$.

On June $1 \&$ 2, 2011, (354) Eleonora was observed with the NASA Infrared TelescopeFacility (IRTF) at Mauna Kea Observatory using the SpeX instrument in its low resolution or asteroid mode (Rayner et al., 2003). The particulars of the Eleonora observations are given in Table 2. Table 3 lists the standard stars used in the observations. The observations of the local standard stars $\left(\operatorname{Std}^{*} 1 \& \operatorname{Std} * 2\right)$ were taken between the sets of Eleonora observations. The solar calibrated standard stars were observed to correct the Eleonora spectra for any slopes introduced by non-solar local standard stars.

Table 2. Observing circumstances for (354) Eleonora.

\begin{tabular}{|l|l|l|l|l|l|l|l|}
\hline Date & Start & End & Sets & Total & R.A. (h) & Dec $\left(^{\circ}\right)$ & $\beta\left(^{\circ}\right)$ \\
\hline $6 / 1 / 11$ & $6: 48$ UT & $12: 36$ UT & $11^{\text {a }}$ & $150^{\mathrm{c}}$ & $16^{\text {hr }} 27^{\mathrm{m}}$ & $+5^{\circ} 50^{\prime}$ & 9.73 \\
\hline $6 / 2 / 11$ & $8: 19$ UT & $12: 19$ UT & $5^{\text {b }}$ & $76^{\mathrm{c}}$ & $16^{\text {hr }} 26^{\mathrm{m}}$ & $+5^{\circ} 49^{\prime}$ & 9.80 \\
\hline
\end{tabular}
a. The 11th set was not used because of insufficient extinction coverage.
b. The 5th set was not used because of insufficient extinction coverage.
c. The first spectrum in each set was omitted because of image persistence on the detector array.

Table 3. Standard stars for Eleonora observations.

\begin{tabular}{|l|l|l|l|l|l|l|}
\hline STAR & AKA & Local/calib. & R.A. (h) & Dec $\left(^{\circ}\right)$ & Type & V mag. \\
\hline SAO121593 & HD 148165 & Std $\# 1$ & 16.43 & 5.9 & G5 & 9.15 \\
\hline SAO121599 & HD 148243 & Std $\# 2$ & 16.44 & 5.6 & G0 & 9.21 \\
\hline SAO120107 & HD 120050 & Solar calib. & 13.78 & 6.0 & G5III & 9.26 \\
\hline SAO141267 & HD 149616 & Solar calib. & 16.58 & 0.4 & G0 & 8.23 \\
\hline
\end{tabular}


The local standard star observations were used to derive atmospheric extinction coefficients (starpacks). Starpacks were derived from all local standard star observations, and subsets of observations (e.g., the pair of sets bracketing a set of Eleonora observations). Prior to derivation of the starpacks, individual sets of standard star observations were compared to a reference set to detect and compensate for displacement of the dispersed spectrum on the detector array due to slight flexure of the instrument. Although the offset was typically at the subpixel level, failure to correct it produces an interference pattern which is especially pronounced in the $\sim 1.9 \mu \mathrm{m}$ atmospheric water vapor absorption feature (Gaffey et al., 2002). This mimics additional noise in the spectral interval of the water feature, but in fact is an interfering signal which can be removed.

Individual asteroid observations are reduced by one or more starpacks. The channel offset is determined for each individual observation prior to processing. The starpack is used to compute the standard star flux curve at the same airmass as the asteroid observation, and the asteroid flux curve is then divided by the standard star flux curve to get the asteroid/standard star flux ratio. Since the standard stars are close to solar type, the ratio is close to a spectral reflectance curve. Fig. 5 shows the average normalized spectral flux curve for (354) Eleonora relative to standard star \#1.

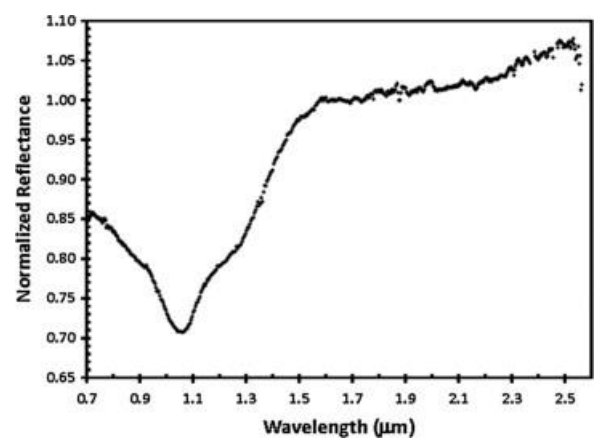

Fig. 5. Average spectra flux of (354) Eleonora relative to standard star \#1 (SAO121593) normalized to $1.7 \mu \mathrm{m}$. Several data points near 2.374 and $2.459 \mu \mathrm{m}$ have been deleted due to an artifact produced by a crack in the detector array. Other than those points, the average includes all data points from the suite of observations.

This is a very typical spectrum of an olivine-dominated assemblage, with only a broad shallow pyroxene feature longwards of $\sim 1.6 \mu \mathrm{m}$. The analysis of this spectrum will be discussed in a subsequent section.

Fig. 6A-E compares the sequential rotational aspects to the overall average. The variations with rotation form as consistent pattern as seen in Fig. 7A which shows the relative reflectance of Eleonora at $0.8 \mu \mathrm{m}$ versus rotation. The mean values for each rotational set are joined by the heavy black line. There is generally good agreement between the means for sets obtained at different times but at similar rotational aspects. Fig. 7B expands the interval around the Rot $=0.6$ "peak", which shows a pattern for the mean values that lend credence to the variation. Fig. $6 \mathrm{~F}$ shows the spectra of the rotational aspects with the highest and lowest reflectance at $0.8 \mu \mathrm{m}$. 

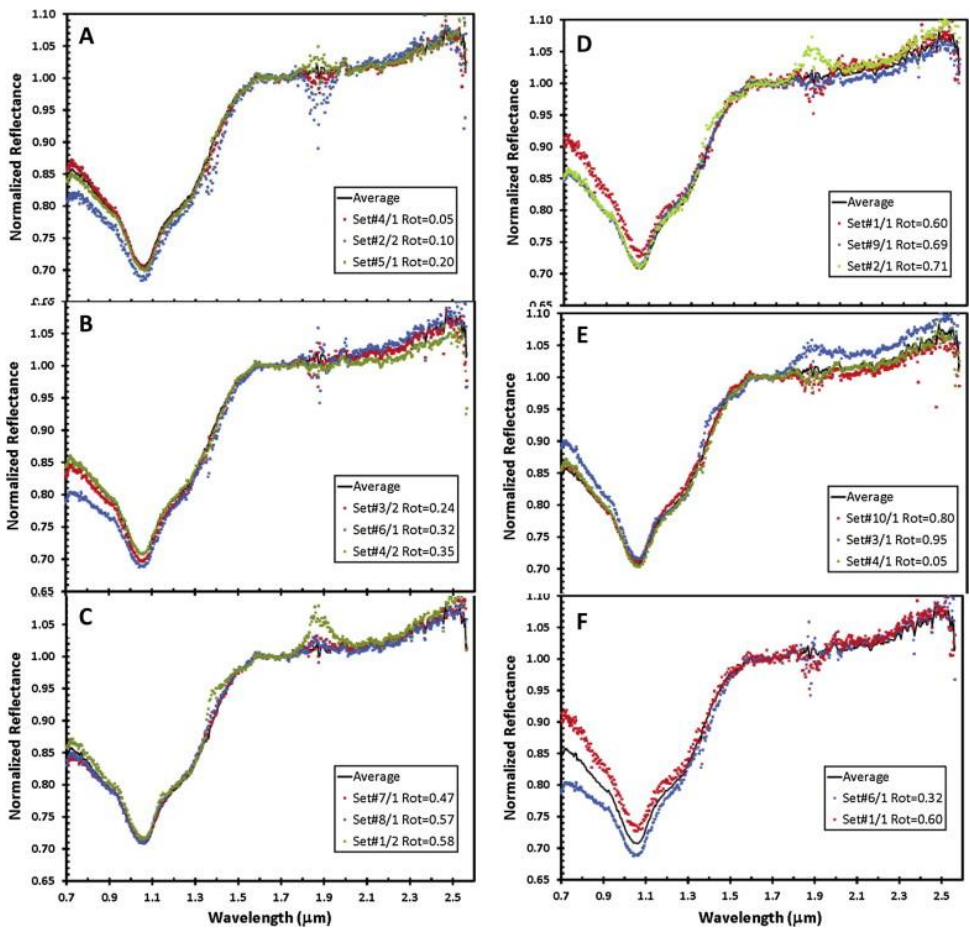

Fig. 6. (A-E) Compare the average spectra obtained at fourteen sequential rotational aspects. Spectra have been normalized at $1.7 \mu \mathrm{m}$. Each set is identified by the code "Set\#N/M", where "M" is either 1 or 2 indicating June 1 or June 2, and "N" is the set of observations on that night. There were ten sets of observations on June 1 and four sets on June 2 . The rotational aspect was calculated for a rotational period of $4.2772 \mathrm{~h}$ (Drummond et al., 1991, Magnusson, 1990, Kaasalainen et al., 2002) with phase $=0.0$ at $0 \mathrm{~h} \mathrm{UT,} \mathrm{June} 1,2011$. Several of the data sets show poorly corrected water vapor absorption features near 1.4 and/or $1.8 \mathrm{~mm}$. The anomalous spectrum beyond $\sim 1.8 \mu \mathrm{m}$ for "Set $\# 3 / 1$ Rot $=0.95$ " is currently unexplained. (F) Shows the spectra with the maximum and minimum reflectances at $0.8 \mu \mathrm{m}$ compared to the overall average.
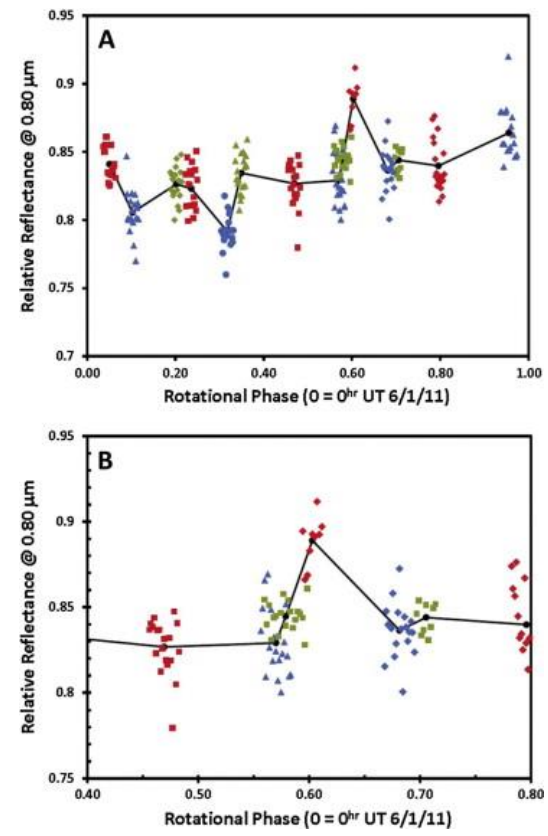

Fig. 7. (A) Relative reflectance of (354) Eleonora at $0.8 \mu \mathrm{m}$ versus rotational aspect. The heavy black line joins the means for each data set. (B) Relative reflectance for rotational aspects near the Rot $=0.6$ "peak". The colors of the symbols follow the same pattern as in Fig. 6. 
These spectra show definite rotational variations, although the $1 \mu \mathrm{m}$ olivine feature is present at all rotational aspects. However, these variations cannot explain the difference in the several short wavelength spectral data sets. Fig. 8 compares the 24-filter, SMASS-I, SMASS-II and S3OS2 data to the average (354) Eleonora spectrum from the present work. The new SpeX data has been scaled to match the 0.73$1.00 \mu \mathrm{m}$ filters in the 24-filter data set. The slightly steeper slope of the band in the 24-filter data in the overlap region is within the range of variation seen in the SpeX data discussed above. It was not possible to match the CCD data to the new SpeX data in the overlap region.

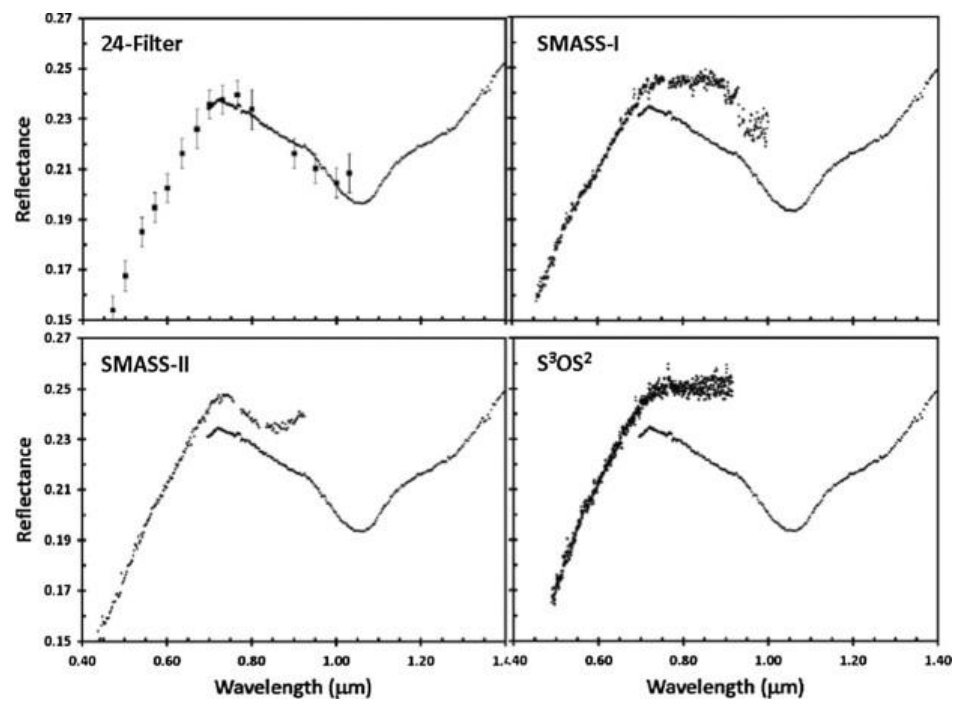

Fig. 8. Previous 24-filter and CCD reflectance spectra of (354) Eleonora compared to the new SpeX data in the region of the $1 \mu \mathrm{m}$ absorption feature. Only the 24-filter data has been scaled to the SpeX data in the overlapping wavelength region.

As Fig. 8 shows, none of the CCD data sets are good matches to the overlapped spectral interval of the SpeX data. The SMASS-I spectrum is flat across the $\sim 0.7-0.9 \mu \mathrm{m}$ interval, where the SpeX and 24-filter data (and to lesser extent the ECAS data) show a $\sim 10 \%$ decrease in reflectance. The SMASS-II spectrum initially shows a reflectance decrease in the $\sim 0.7-0.85 \mu \mathrm{m}$ which parallels the SpeX curve, but then bottoms out and turns upward where the SpeX curve is still decreasing. The S3OS2 spectrum is flat (or slightly increasing) across the overlapping wavelength interval with no indication of the absorption feature. The diversity seen in CCD spectra would lead to very different interpretations of the surface mineralogy of Eleonora and its evolutionary history.

The discrepancy must lie with the CCD data, as demonstrated in Fig. 9 which compares the NIR spectra of Eleonora obtained by the various observers to the new spectrum obtained in the present work. The differences between the new spectrum (dashed line) and the previous NIR spectra, which were obtained with three different instruments, lay primarily in the overall slope and the depth of the $1 \mu \mathrm{m}$ feature. These differences are broadly comparable to the rotational spectral variations for Eleonora discussed above. Some of the slope differences also probably arise from the use of "slightly non-solar" standard stars (this is discussed in more detail below). In contrast to interpretations of the CCD data, mineralogical interpretations of all the NIR spectra - excepting the short SMASSIR spectrum - would be quite consistent with an olivine dominated assemblage possibly with a trace of pyroxene. Although the SMASSIR spectrum is too short to derive such an interpretation, it is certainly consistent with it. 


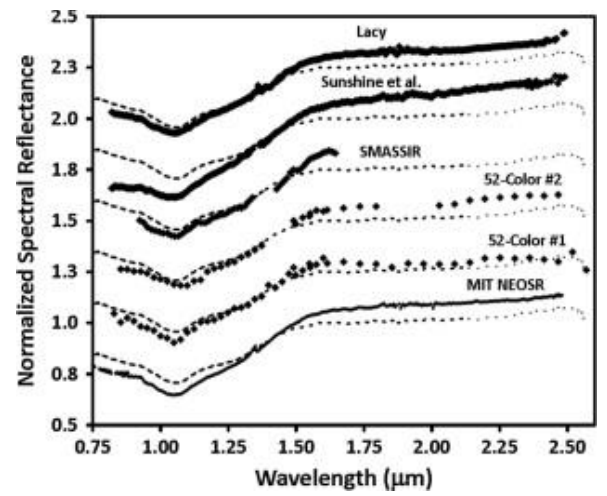

Fig. 9. Same data as shown in Fig. 3 except the new SpeX data (dashed line) has been included for comparison. Spectra have been offset vertically by steps of 0.25 for clarity.

We are thus led to the conclusion that the differences between the short wavelength spectra is not due to rotational spectral variations produced by a longitudinally heterogeneous surface on Eleonora.

\subsection{Testing for latitudinal compositional heterogeneity}

Because the rotation axis of (354) Eleonora is tilted relative to its orbital plane, observations in different portions of its orbit result in observations of different sub-Earth (sub-observer) latitudes on the body. Thus if the body has a north-south asymmetry in its surface composition, observations at different subEarth latitudes (SEL) would result in different spectra. Table 4 lists the observational parameters for the data sets being considered here.

Table 4. Observing circumstances for (354) Eleonora spectra.

\begin{tabular}{|l|l|l|l|l|l|l|l|}
\hline Data set & Reference & MM/DD/YR & $\mathbf{R A}^{\mathbf{a}}$ & $\mathbf{D e c}^{\mathbf{a}}$ & $\boldsymbol{r}(\mathbf{A U})^{\mathbf{a}}$ & Phase $^{\mathbf{a}}$ & $\mathbf{S E L}^{\mathbf{b}}$ \\
\hline ECAS-1 & Zellner et al. (1985) & $8 / 24 / 1979$ & 306.2 & -15.4 & 3.105 & 8.2 & -37.6 \\
\hline ECAS-2 & Zellner et al. (1985) & $9 / 30 / 1980$ & 35.1 & -11.1 & 2.950 & 10.2 & -35.9 \\
\hline 24-filter & Chapman and Gaffey (1979) & $4 / 25 / 1973$ & 183.5 & 21.4 & 2.547 & 16.2 & 49.0 \\
\hline SMASS-I & Xu et al. (1995) & $3 / 11 / 1992$ & 226.9 & 6.4 & 2.605 & 18.3 & 27.5 \\
\hline SMASS-II & Bus and Binzel, 2002a, Bus and Binzel, 2002b & $10 / 27 / 1995$ & 129.9 & 7.2 & 2.534 & 22.9 & 45.2 \\
\hline SMASS-II & Bus and Binzel, 2002a, Bus and Binzel, 2002b & $2 / 22 / 1997$ & 251.9 & -3.4 & 2.720 & 21.2 & 7.0 \\
\hline S3OS ${ }^{2}$ & Lazzaro et al. (2004) & $8 / 30 / 1998$ & 331.6 & -16.8 & 3.118 & 3.2 & 52.7 \\
\hline 52-color \#1 & Bell et al. (1988) & $8 / 2 / 1984$ & 339.6 & -12.4 & 3.119 & 8.4 & -59.7 \\
\hline 52-color \#2 & Bell et al. (1988) & $12 / 22 / 1985$ & 53.4 & -7.1 & 2.725 & 15.4 & -21.3 \\
\hline SMASSIR & Burbine and Binzel (2002) & $2 / 9 / 1997$ & 248.2 & -4.0 & 2.705 & 20.5 & 10.7 \\
\hline Sunshine & Sunshine et al. (2007) & $4 / 15 / 2002$ & 284.1 & -5.1 & 2.906 & 19.9 & -23.1 \\
\hline MIT NEOSR & DM02 & $8 / 8 / 2011$ & 241.5 & -1.6 & 2.888 & 19.9 & 16.4 \\
\hline Lacy & Gietzen et al. (2012) & $5 / 30 / 2006$ & 210.1 & 15.1 & 2.671 & 17.2 & 37.8 \\
\hline This work & This paper & $6 / 1 \& 2 / 2011$ & 246.8 & 5.8 & 2.810 & 9.7 & 9.1 \\
\hline
\end{tabular}

a. Observational parameters calculated using the JPL Horizons system ephemeris program.

b. Sub-Earth latitude (SEL) was computed for the Kaasalainen et al., (2002) pole position at ecliptic longitude $=356^{\circ}$ and ecliptic latitude $=+20^{\circ}$. 
c. The SMASS-II CCD spectrum is an average of data obtained on two different nights, so the effective geometry would be different than either night.

If this mechanism is responsible for the differences in the short wavelength spectra, then observations at similar sub-Earth latitudes should produce similar spectra. Fig. 10 compares the $\mathrm{S}^{3} \mathrm{OS}^{2} \mathrm{CCD}$ spectrum to the 24-filter spectrum, both of which were obtained at high northern latitudes ( $53^{\circ}$ and $49^{\circ}$, respectively). These are the most disparate of the short wavelength spectra, yet they were obtained at virtually the same sub-Earth latitudes. The SMASS-II Spectrum is an average of observations taken at SEL $=47^{\circ} \& 7^{\circ}$. However its reflectance curve longwards of $\sim 0.7 \mu \mathrm{m}$ does not resemble the curves of the 24-filter data $\left(\mathrm{SEL}=+49^{\circ}\right)$, the Lacy spectrum $\left(\mathrm{SEL}=+38^{\circ}\right)$ or the new SpeX spectrum $\left(\mathrm{SEL}=+9^{\circ}\right)$ or an average of these data. Thus the possibility that the diversity on the CCD spectra is due to latitudinal compositional variations on the surface of (354) Eleonora can be eliminated.

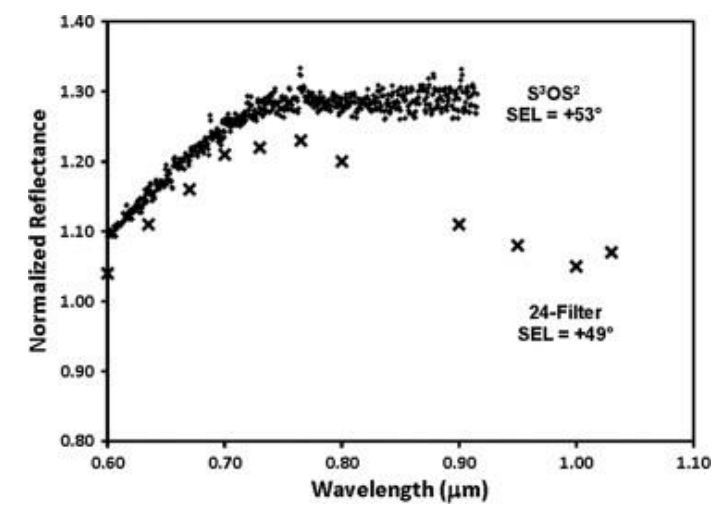

Fig. 10. Comparison of spectra taken at similar sub-Earth latitudes.

\subsection{Testing for spectral phase effects}

The observational phase angles for the Eleonora spectra discussed here vary from $3.2^{\circ}$ to $22.9^{\circ}$. Gradie and Veverka (1986) demonstrated that for mafic silicate assemblages (they used the spectral parameters of the L6 chondrite Bruderheim), the spectral curve shortwards of the $\sim 0.7 \mu \mathrm{m}$ peak steepened (reddened) and the $\sim 1 \mu \mathrm{m}$ absorption feature deepened by $\sim 12 \%$ from $4^{\circ}$ to $60^{\circ}$ phase. Gaffey (1997) noted that the $1 \mu \mathrm{m}$ feature in the spectrum of (4) Vesta was weaker at phase $=4^{\circ}$ than at phase $=17^{\circ}$ by the amount predicted by the Gradie and Veverka (1986) results. Reddy et al. (2012) showed that the depth of the $\sim 1$ $\mu \mathrm{m}$ absorption feature (Band I) in the spectrum of Asteroid (4) Vesta increased linearly from $\sim 30.1 \%$ at phase $=4.9^{\circ}$ to $\sim 34.6 \%$ at phase $=24^{\circ}$.

Although the S3OS2 spectrum was obtained at the smallest phase angle $\left(3.2^{\circ}\right)$, none of the calibration work suggests that the depths of the mafic silicate features approach zero as the phase angle approaches zero. By contrast, both SMASS-II spectra were obtained at the highest phase angles $\left(21.2^{\circ} \& 22.9^{\circ}\right)$, and they only exhibit a very weak (and atypical feature). There is no clear pattern of variation in band depth among the NIR spectra as a function of phase angle.

Thus the diversity among the CCD spectra and their mismatch to the other spectral data sets cannot be explained by the differences in the observational phase angle. 


\subsection{Testing for spectral temperature effects}

Temperature induced spectral changes in the VNIR spectral region are particularly significant for the diagnostic crystal field features. The wavelength position and width of these features are parameters used in quantitative analysis of spectral data (e.g., Adams, 1974, King and Ridley, 1987, Cloutis and Gaffey, 1991, Sunshine and Pieters, 1998). The positions of these features are determined by the relative energies of the electron orbitals of a transition metal cation (typically $\mathrm{Fe} 2+$ ) in a coordination site (commonly octahedral in silicates) in the mafic mineral species. The dimensions of the coordination site increase and decrease with increasing and decreasing temperature, changing the relative energies of the electron orbitals of a transition metal cation, causing the spectral features to move. In a similar fashion, higher temperatures cause more energetic lattice vibrations increasing the width of the absorption features.

The effects of temperature on VNIR reflectance spectra have been studied by a number of investigators (e.g., Roush, 1984, Roush and Singer, 1986, Lucey et al., 1998, Moroz et al., 2000, Hinrichs and Lucey, 2002, Sunshine et al., 2007, Reddy et al., 2011a, Reddy et al., 2011b). The broad $1 \mu \mathrm{m}$ feature in olivine is sensitive to temperature (e.g., Fig. 4 in Lucey et al., 1998; Fig. 2 in Hinrichs and Lucey, 2002). In Fo89 olivine, the feature broadens linearly with increasing temperature from a full width at half maximum (FWHM) of $\sim 0.42 \mu \mathrm{m}$ at $83 \mathrm{~K}$ to a FWHM of $\sim 0.78 \mu \mathrm{m}$ at $448 \mathrm{~K}$ (Roush, 1984 shown in Fig. 6 of Lucey et al., 1998). However, over the same temperature range, the depth of the olivine absorption band remains nearly constant (Fig. 4 in Roush, 1984; Fig. 6 in Hinrichs and Lucey, 2002).

The effective temperatures for each of the observations were calculated by several different models including the equation from Burbine et al. (2009). While the absolute values differ between the models, the relative temperatures and the range of temperatures are very similar. The coolest surfaces observed (52-color Set\#1 \& S3OS2) are about $20^{\circ}$ cooler than the warmest surfaces observed (SMASS-II/95, 24filter \& SMASS-I). With a change in the FWHM of the olivine feature of $\sim 0.001 \mu \mathrm{m} / \mathrm{K}$, this $20 \mathrm{~K}$ difference would correspond to an increase of $\sim 0.02 \mu \mathrm{m}$ in the FWHM of the olivine feature in the spectra from the "warmer" observations compared to the spectra from the "cooler" observations. This should be detectable in well calibrated spectral data. However, temperature effects can explain neither the diversity of the CCD spectra or the discrepancy in the $1 \mu \mathrm{m}$ absorption feature between the CCD and other spectra.

\subsection{Testing for spectral effects of asteroid shape}

Gradie and Veverka (1981, Fig. 10) showed that for an ellipsoidal body with an equatorial axial ratio of 3 $(a / b=3$ where " $a$ " is the longest equatorial dimension and " $b$ " is the shortest equatorial dimension) and surfaced with a mafic silicate assemblage (the L6 Chondrite Bruderheim) the depth of the $1 \mu \mathrm{m}$ absorption feature varied $\sim 5 \%$ between the end and side of the body when observed at phase $=3^{\circ}$. The differences decrease with increasing phase angle to $\sim 3 \%$ at phase $=30^{\circ}$ and $\sim 1.5 \%$ at phase $=60^{\circ}$. The magnitude of these shape related spectral variations would be zero for a sphere or oblate ellipsoid and would increase with increasing a/b ratio.

Fig. 11 shows a selection of lightcurves obtained for (354) Eleonora at various sub-Earth latitudes. The amplitudes of the lightcurves decrease away from the equatorial viewing geometry as expected. The maximum amplitude observed at $\mathrm{SEL}=+2^{\circ}$ is $\sim 30 \%$. Moreover, the lightcurve shapes are relatively sinusoidal and do not exhibit any abrupt changes in brightness that would be observed if large flat facets existed on the surface. Such facets would have significant effects on the viewing geometry (incidence and emission angles of the surface units) as they rotated into and out of view. 


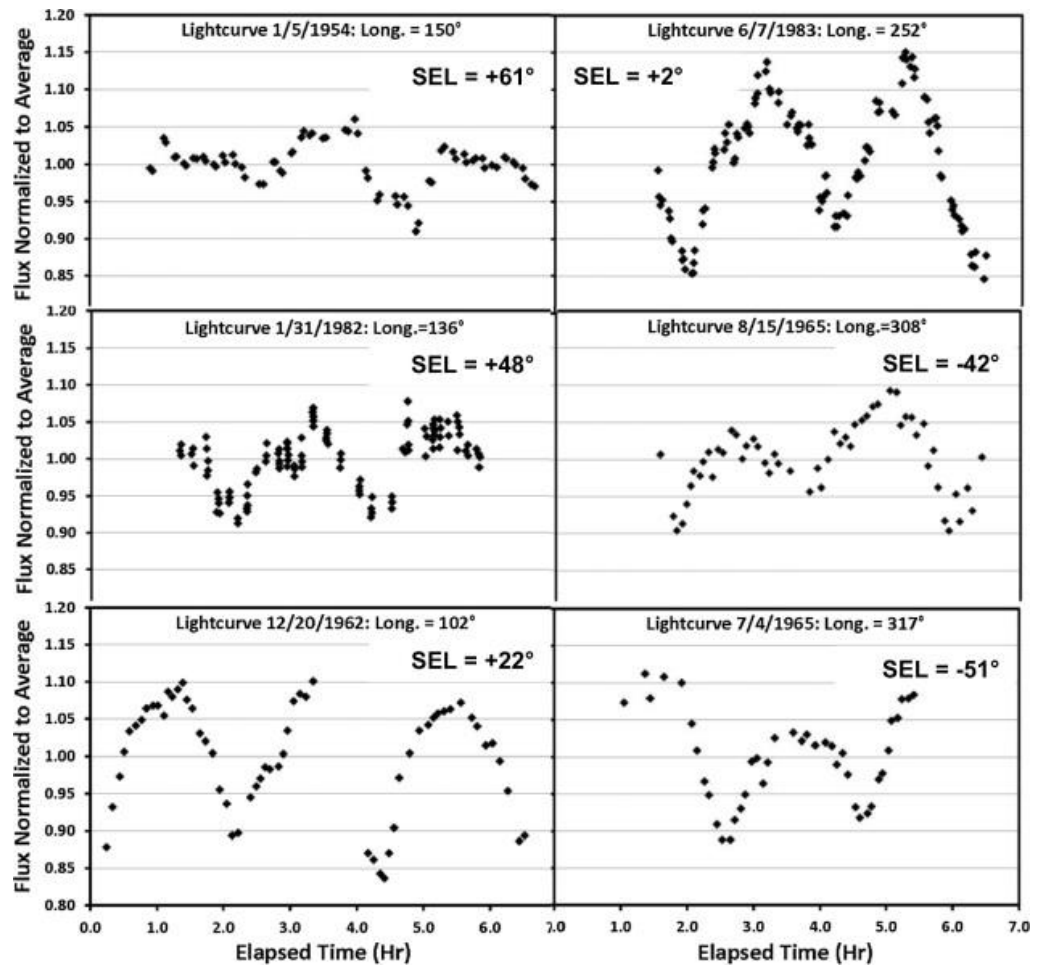

Fig. 11. Lightcurves for (354) Eleonora for six different viewing geometries. The sub-Earth latitude (SEL) on the asteroid is computed for the Kaasalainen et al. (2002) pole orientation at ecliptic longitude $=356^{\circ}$ and ecliptic latitude $=+20^{\circ}$. Lightcurves from: 1/5/1954 - Groeneveld and Kuiper (1954); 1/31/1982 - Piironen et al. (1985); 12/20/1962 - Chang and Chang (1963); 6/7/1983 - Burchi et al. (1985); 8/15/1965 - Zappalà et al. (1979); 7/4/1965 - Zappalà et al. (1979). Digital data from Lagerkvist and Magnusson (2011).

Kaasalainen et al. (2002) used the lightcurve data to derive a shape model for (354) Eleonora. They concluded that Eleonora was "quite a regular body" with an $\mathrm{a} / \mathrm{b}=1.2$. Although it is not possible to establish the lightcurve phase of the spectral observations considered here - and hence to constrain the shape effect on each spectrum - the magnitude of the effect for an object with such a low $\mathrm{a} / \mathrm{b}$ ratio would be minimal. Moreover, even if the axial ratio was much higher, the effect would not eliminate the $1 \mu \mathrm{m}$ feature. Thus, the differences between the CCD spectra and with the other spectra cannot be reconciled by invoking shape-related spectral variations.

\section{Discussion}

There are two issues to address: (a) why do the CCD spectra of (354) Eleonora differ from each other, and (b) why do the CCD spectra differ from the other spectral data (i.e., ECAS, 24-filter), and from the seven sets of NIR spectra? Ignoring for the moment the mismatch between the $1 \mu \mathrm{m}$ feature in the CCD spectra and in the other spectral data sets, how significant is the mismatch between the three CCD data sets? Fig. 12 compares the three CCD spectra and the SpeX data with the closest match. The SMASS-II spectrum, which exhibits a weak but well defined absorption feature centered near $0.85 \mu \mathrm{m}$, is clearly discordant with the SMASS-I and S3OS 2 spectra which are essentially flat across the $\sim 0.75-0.90 \mu \mathrm{m}$ spectral interval. The SMASS-I spectrum shows a drop off into a putative absorption band longwards of $\sim 0.90$ $\mu \mathrm{m}$, but since the S3OS2 spectrum does not include this spectral interval, one could plausibly argue that up to $\sim 0.90 \mu \mathrm{m}$, these spectra are consistent, although the two spectra would lead to quite different compositional interpretations. The Sunshine et al. (2007) SpeX spectrum generally resembles the SMASS-I spectrum in the overlap region, with a relatively flat reflectance across the $\sim 0.84-0.94 \mu \mathrm{m}$ spectral interval then dropping off into a feature centered near $1.05 \mu \mathrm{m}$. Could whatever conditions 
produced the nearly flat short wavelength reflectance in the Sunshine et al. (2007) spectrum be operating on the SMASS-I and S3OS2 CCD spectra?

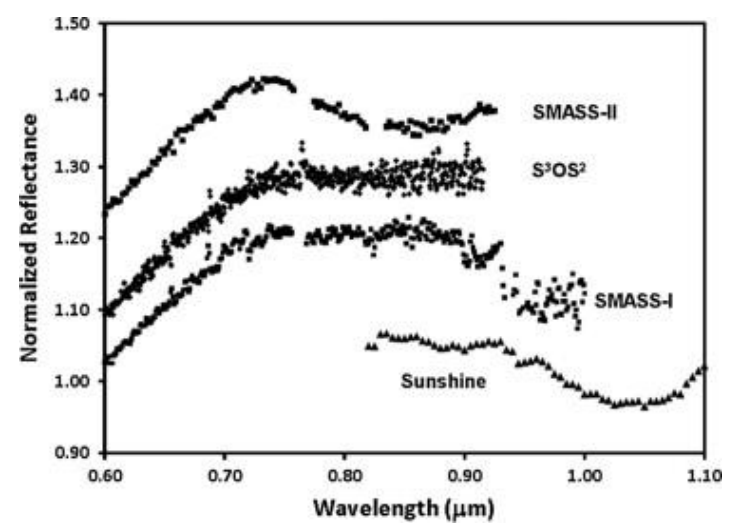

Fig. 12. Comparison of $1 \mu \mathrm{m}$ spectral band interval for all CCD data sets and the SpeX data set from Sunshine et al. (2007). Spectra have been offset vertically for clarity.

The Sunshine et al. (2007) spectrum has the steepest reflectance curve of the NIR data sets (Fig. 9). There are several possible causes of the spectral slope differences among the NIR data, but the most likely is the effect of using somewhat non-solar standard stars. To check this possibility, the several NIR data sets have been interpolated using a cubic spline interpolation to the SpeX wavelengths used in the present study, and then divided by the average spectrum from the current study (Fig. 13).

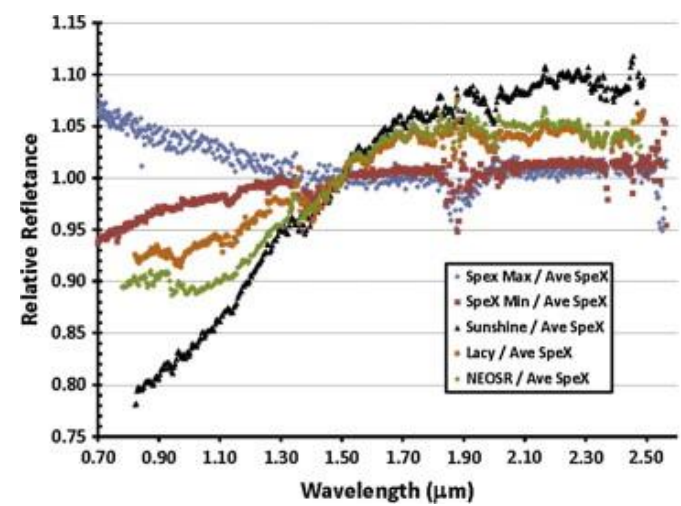

Fig. 13. NIR reflectance spectra of (354) Eleonora relative to the overall average from the present study. The red and blue symbols represent the upper and lower limits for the spectral variations observed in the present study (Fig. 6).

The primary standard star (SAO 121593: Table 3) used in the present study was calibrated through SAO 129107 and BS/HR 5996 (HD144585/SAO 159706; a Hardorp (1978) Type 3 "spectrum very close to solar" star) appears to be slightly cooler than a true solar analog. All of the other NIR spectra, are consistent with standard stars somewhat hotter than the standard star used in this study. To a first approximation stars emit as black bodies. The normalized ratios of black bodies with different temperatures form smooth curves. The spectral ratios shown in Fig. 13 display such smooth curves and are consistent with the standard star(s) used for the Sunshine et al. (2007) observations being hotter than the standard stars used for the Lacy and NEOSR observations which in turn were hotter than the standard star used in the present study.

Relatively few accurately calibrated NIR standard stars with appropriate magnitudes are available for asteroid observers using the SpeX instrument. (This is an issue of long standing, but sufficient funding 
and telescope time has not been available to carry out a dedicated program to calibrate an appropriate network of standard stars.) In most cases we are satisfied with "close to solar" rather than requiring "strictly solar" standards. The major effect of using "close to solar" standards is that they introduce changes in the overall spectral slopes. Except for situations where the standard star is wildly nonsolar, this does not constitute a significant problem because the spectral slope is removed or isolated before analysis by any of the quantitative analysis techniques (e.g., MGMFIT - Sunshine et al., 2007; BAR \& Band Center analysis - Gaffey et al., 2002, Burbine et al., 2009, Dunn et al., 2010). Thus the overall spectral slope plays little or no role in the analysis of such spectra.

The question remains as to whether the SMASS-I and S3OS2 spectra could be the result of observations using hotter standard stars which would flatten the short wavelength wing of the olivine absorption feature. This is unlikely because as a general rule, the calibration of VNIR $(\lambda<\sim 1 \mu \mathrm{m})$ standard stars (e.g., Hardorp, 1978, Hardorp, 1980, Tedesco et al., 1982, Chapman et al., 1973) is much better than the calibrations for NIR $(\lambda \sim .8-2.5 \mu \mathrm{m})$ standard stars.

However the main reason for excluding hotter standard stars as the cause of the "flat" segments in the SMASS-I and S3OS2 spectra is that if that were the case, the slope of the spectrum shortwards of the absorption feature should also be steepened, which is clearly not the case (Fig. 2). In fact hotter standard stars would produce a greater steepening at the visible wavelengths than at the NIR wavelengths.

\section{Conclusion with respect to the $\mathrm{CCD}$ data}

Thus we are unable to identify a viable mechanism to reconcile the SMASS-II and SMASS-I \& S3OS2 CCD spectra with each other, or with the 24-filter, ECAS, or NIR spectra. At the present time, we are left with the unpalatable conclusion that the CCD spectra for (354) Eleonora are unreliable. The more important question is whether this a problem unique to (354) Eleonora. Note that Eleonora was selected for reasons unrelated to the differences in the CCD spectra (e.g., Gaffey et al., 1993).

It will be important to determine how many other objects mutually observed by SMASS-I, SMASS-II and S3OS2 exhibit similar discrepancies. Such discrepancies would lead to very different conclusions about the compositions of these bodies. On the more positive side, such differences may flag objects with real surface variegation. If such disagreements are the results of "bad" data, it will be important to identify criteria for distinguishing such "bad" data within the survey data sets.

\section{Analysis of the current data set}

Fig. 14 shows the VNIR reflectance spectrum of (354) Eleonora merged from the average new SpeX data and the previous 24-filter data. The spectrum exhibits a broad olivine-type feature centered near $1.05 \mu \mathrm{m}$ and a broad shallow feature between $\sim 1.6$ and $2.5 \mu \mathrm{m}$. The SpeX wavelength interval encompasses the full width of the broad $1 \mu \mathrm{m}$ absorption feature, so that subsequent mineralogical analysis will focus almost entirely on the SpeX spectrum. 


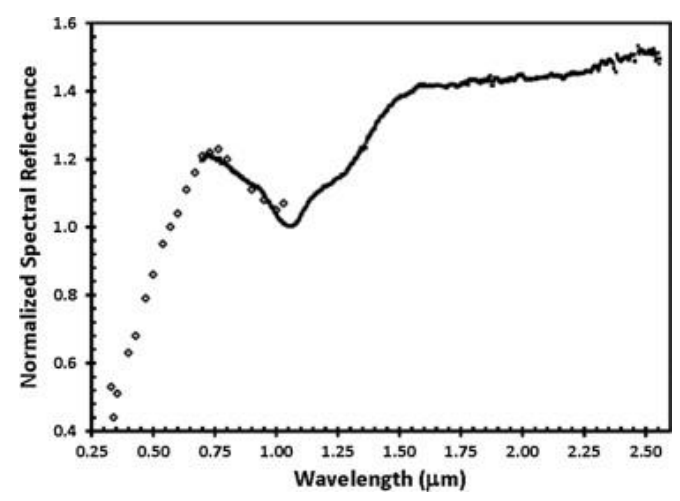

Fig. 14. Reflectance spectrum of (354) Eleonora from average SpeX data from present investigation along with the 24-filter data from Chapman and Gaffey (1979). The 24-filter data was normalized at $0.57 \mathrm{~mm}$ and the SpeX data was scaled to the 24-filter data in the overlapping wavelength interval.

Fig. 15 shows the SpeX data after removal of linear continuum segments fitted tangent to the peaks in the reflectance curve. The central wavelengths of Bands I and II were determined by fitting several polynomials to the channels near the band minima. The Band I center was determined using 26 channels centered on the continuum-removed band minimum for both the average of all SpeX data and for the averages of the individual rotational segments. The Band I center for the average spectrum and for the averages of the individual rotational segments was $1.062 \pm 0.002 \mu \mathrm{m}$. (Estimated uncertainty based on the standard deviation of the average from the fourteen individual rotational aspects. The range of values for the Band I center for those aspects was from 1.059 to $1.066 \mu \mathrm{m}$.) The spectral parameters and derived properties for the average spectrum of 354 Eleonora from our observations is shown in Table 5.

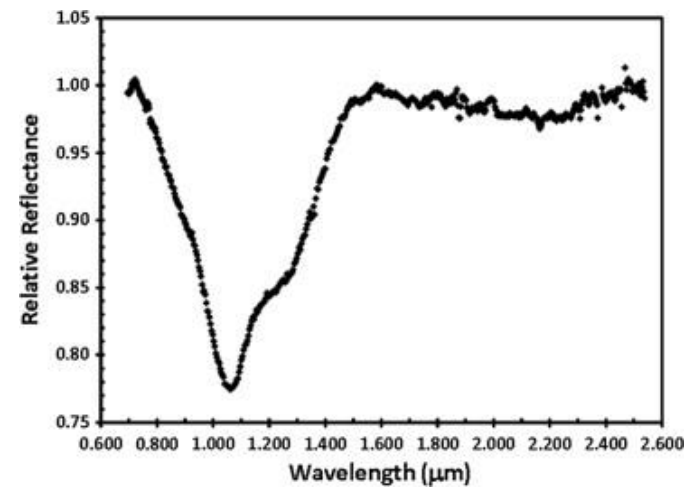

Fig. 15. Average SpeX spectrum of (354) Eleonora after removal of linear continuum segments. 
Table 5. Parameters for (354) Eleonora.

\begin{tabular}{|l|l|}
\hline Parameter & Value \\
\hline Band I center $^{\mathrm{a}}$ & $1.062 \pm 0.002 \mu \mathrm{m}$ \\
\hline Band II center $^{\mathrm{a}}$ & $\sim 2.13 \mu \mathrm{m}$ \\
\hline Band Area Ratio $^{\mathrm{a}}$ & $0.112 \pm 0.006$ \\
\hline Surface temperature & \\
\hline Temperature correction to Band I center & \\
\hline Band I depth & \\
\hline FWHM $^{\mathrm{a}}$ & $+0.004 \mu \mathrm{m}$ \\
\hline Albedo $^{\mathrm{d}}$ & $22.5 \%$ \\
\hline Olivine composition $^{\mathrm{e}}$ & $0.401 \mathrm{~mm}^{\mathrm{a}}$ \\
\hline
\end{tabular}

a. Measured from our VNIR spectra.

b. Calculated using equation from Burbine et al. (2009) and the observational circumstances (Table 2).

c. Temperature correction using Eq. (3) from Sanchez et al. (2014).

d. Albedo from Small Bodies Node of Planetary Data System.

e. Olivine compositions calculated from temperature corrected band I center using calibrations of King and Ridley, 1987, Reddy et al., 2011a, Reddy et al., 2011b and Dunn et al. (2010), respectively.

The ratio of Band II area to Band I (Band Area Ratio; BAR) is $0.112 \pm 0.006$. Assuming that the Band II feature is entirely due to orthopyroxene, this corresponds to $9.8 \%$ orthopyroxene using the calibration from Gaffey et al. (2002) $(\mathrm{OPX} /(\mathrm{OPX}+\mathrm{OL})=0.417 * \mathrm{BAR}+0.052)$ or to $8.7 \%$ orthopyroxene using a calibration derived from the entire suite of orthopyroxene-olivine mixtures measured by Cloutis et al. (1986) $(\mathrm{OPX} /(\mathrm{OPX}+\mathrm{OL})=0.4305 * \mathrm{BAR}+0.0383)$. This would overestimate the pyroxene abundance if the underlying $\sim 1.5-2.5 \mu \mathrm{m}$ curve for the olivine was not linear but had a slight positive curvature as is seen in some laboratory olivine spectra.

Although the width of the $1 \mu \mathrm{m}$ olivine feature is very temperature sensitive, the central wavelength is less so (Singer and Roush, 1985, Roush and Singer, 1986, Lucey et al., 1998, Moroz et al., 2000, Hinrichs and Lucey, 2002). Using the temperature equation of Burbine et al. (2009) we derived a surface temperature of $160 \mathrm{~K}$ at the epoch of the observations. We have applied the olivine band center temperature correction derived by Sanchez et al. (2014) and Reddy et al., 2011a, Reddy et al., 2011b which shifts the band center for 354 Eleonora to $1.066 \mu \mathrm{m}$ and calculated the olivine composition from this corrected band central wavelength. The average composition of the Eleonora olivine can be estimated from a calibration derived from the King and Ridley (1987) measurements of olivine band center versus molar composition (Fo = 1825.8-1655.7 Center) or from an expanded set of band position measurements

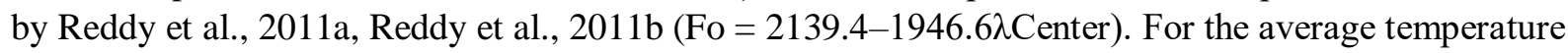
corrected Band I center $(1.066 \mu \mathrm{m})$, these calibrations indicate compositions of Fo61 and Fo64, respectively. For comparison to the values derived without the temperature correction are Fo67 and Fo72, respectively. Although the Dunn et al. (2010) calibration was derived for the olivine pyroxene mixtures of ordinary chondrites, which exhibit significantly stronger pyroxene features than (354) Eleonora, applying that calibration to this data results in an olivine composition of Fa29.4 (Fo70.6), close to the value derived by the other two calibrations. (We report the results for the Dunn calibration to three significant figures 
only because the differences between different rotational aspects noted below are so small.) The uncertainties in these Fo values based on the standard deviation of the points in the King and Ridley (1987) and the Reddy et al., 2011a, Reddy et al., 2011b calibration sets are $\pm 9 \%$ and $\pm 11 \%$, respectively. The uncertainty in the Fo value estimated for Dunn calibration is estimated to be $\pm 1.4 \%$ (Dunn et al., 2010). The range of band centers for the fourteen rotational aspects ranges from 1.059 to $1.066 \mu \mathrm{m}$, which after a temperature correction of $+0.004 \mu \mathrm{m}$ would correspond to Fo66 \& Fo54, to Fo 70 \& Fo57, and to Fo70.9 to Fo70.3, respectively. Although there is considerable scatter, there is a hint that the Band I center wavelength may be shortest near rotational aspect 0.6 and longest near rotational aspect 0.1 (Fig. 16). The area of Band I also shows significant scatter but appears to be weakest near rotational aspect 0.5 (Fig. 17). For the present, we do not give additional consideration these possible variations.

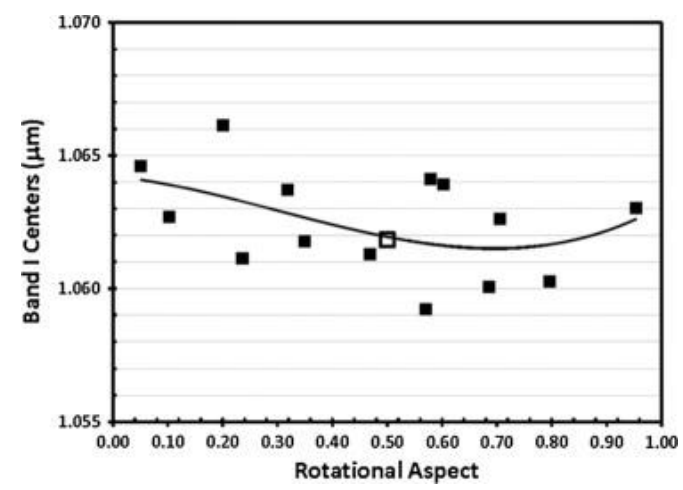

Fig. 16. Band I centers for different rotational aspects of (354) Eleonora. The solid line is a three-term polynomial fit to the distribution. The Band I center for the average Eleonora spectrum is shown by the larger open square.

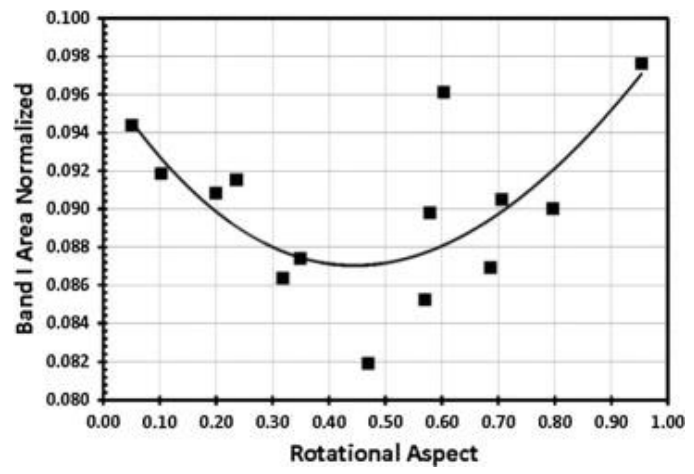

Fig. 17. Normalized area of Band I versus rotational aspect. The solid line represents a third-order polynomial fit to the data.

The putative feature in the $2 \mu \mathrm{m}$ spectral interval is both noisy and weak. Several sets of points across this interval were fitted with polynomial functions and the derived Band II position is $\sim 2.13 \mu \mathrm{m}$. This is in the region of clinopyroxenes measured by Adams (1974) and Cloutis and Gaffey (1991). A calibration based on the Adams (1974) and Cloutis and Gaffey (1991) data would suggest a pyroxene composition of $\sim$ Fs50Wo10 but this is only weakly constrained. (Using only the Band I center, the Dunn calibration would give a pyroxene composition of Fs25, but given that the Band I position in the Eleonora spectrum shows little influence by pyroxene, we do not consider this result reliable.) To the extent that a composition of $\sim$ Fs 50 Wo10 is credible, such a pyroxene composition would indicate an igneous assemblage. 
The width of the olivine feature is a function of three important properties: particle size, olivine composition and temperature. The width of the band in the Eleonora spectrum measured at the half maximum (full width at half maximum; FWHM) is $0.401 \mu \mathrm{m}$. Compared to the scaled Band I curves of laboratory samples of olivine (Fig. 18), the band for (354) Eleonora is very narrow. In particular, it is significantly narrower than the finest size fraction of the olivine powders $(<45 \mu \mathrm{m})$. If the narrow width were due to particle size effects, the surface material of Eleonora would need to have an average particle size significantly smaller than $45 \mu \mathrm{m}$. Based on the particle size distribution in mature lunar regolith, this seems unlikely as the primary cause of the narrow width.

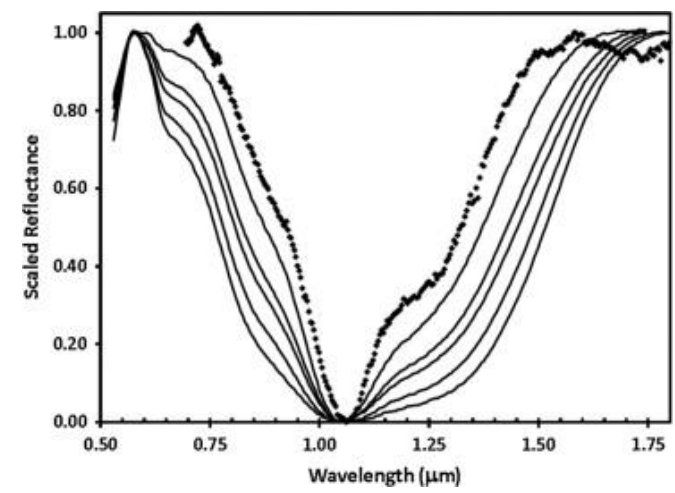

\begin{abstract}
Fig. 18. The continuum-removed scaled absorption band of (354) Eleonora (small symbols) compared to the continuum-removed scaled absorption bands of Green Sand Beach (GSB) olivine (Fo89) for particle sizes $<45 \mu \mathrm{m}$ (inner most solid line), 45-90 $\mu \mathrm{m}$, 90-125 $\mu \mathrm{m}, 125-250 \mu \mathrm{m}$ and $250-500 \mu \mathrm{m}$ (outer most solid line). The olivine samples were purified by hand picking from Green Sand Beach olivine collected at South Point on the Island of Hawaii. Samples were ground and wet sieved to produce the various particle size fractions. The GSB olivine spectra were measured at $\sim 20^{\circ} \mathrm{C}$.
\end{abstract}

Fig. 5 in Lucey et al. (1998) shows the FWHM of the olivine feature measured at $300 \mathrm{~K}$ versus the composition of the olivine based on data from King and Ridley (1987). FWHM varies from $\sim 0.65 \mu \mathrm{m}$ at $\mathrm{Fa} 9$ (Fo91) to $\sim 0.82 \mu \mathrm{m}$ at Fa89 (Fo11). FWHM varies directly with Fe2+ content and inversely with $\mathrm{Mg} 2+$ content of these olivines. The FWHM of the least iron-rich olivine is already substantially greater than that of (354) Eleonora, so the narrowness of the Eleonora feature cannot be primarily due to composition. Moreover, the olivine samples in Fig. 18 are already low iron (Fa9/Fo91), so there would be little room to compositionally narrow the feature further. Using the FWHM values of the spectra of the GSB olivine powders shown in Fig. 18, a rough extrapolation suggests that to get down to the FWHM of Eleonora (0.40), the particle size would need to be $\sim 10 \mu \mathrm{m}$, an unrealistically low value for an impact generated regolith.

Although the surface temperature of (354) Eleonora would have only a small effect on the Band I position (see above), it would have a significant effect on the width of the feature (e.g., Singer and Roush, 1985, Roush and Singer, 1986, Lucey et al., 1998, Moroz et al., 2000, Hinrichs and Lucey, 2002). Lucey et al. (1998) compared an A-type Asteroid (446 Aeternitas) with an expected surface temperature of 120-190 K to olivine spectra measured at temperatures from $83 \mathrm{~K}$ to $448 \mathrm{~K}$ and showed that the spectrum of (446) Aeternitas was generally consistent with a temperature between $83 \mathrm{~K}$ and $173 \mathrm{~K}$.

If the average scaled (354) Eleonora band is overlain in Fig. 4 of Lucey et al. (1998), the Eleonora spectrum is somewhat narrower than the Aeternitas spectrum. Using the plot of FWHM versus temperature (Fig. 6 of Lucey et al., 1998), the FWHM of Eleonora $(0.40 \mu \mathrm{m})$ would correspond to a surface temperature of $\sim 60 \mathrm{~K}$, an unrealistically low temperature. Using the equation for asteroid surface temperature from Burbine et al. (2009) for the June 2011 observations, the heliocentric distance (r) was 
2.810 AU $(4.20 \times 1011 \mathrm{~m})$, giving a surface temperature of $\sim 160 \mathrm{~K}$. Using the relationship between FWHM and temperature, a surface temperature of $160 \mathrm{~K}$ would produce a FWHM of $0.50 \mu \mathrm{m}$, significantly larger than the $0.40 \mu \mathrm{m}$ FWHM measured for (354) Eleonora.

Thus the FWHM for the olivine feature in the (354) Eleonora spectrum is too low to be explained by a reasonable particle size distribution, by an iron poor olivine composition, or a plausible low surface temperature. What can be said with confidence is that the primary mafic silicate mineral on the surface of (354) Eleonora is olivine, and that pyroxene may be present as an accessory phase. The relative intensity of the $1 \mu \mathrm{m}$ absorption feature in the new spectrum of Eleonora is 0.225 , essentially identical to the value (0.24) from the S-asteroid survey (Fig. 1).

Comparison of the spectral albedo curve of (354) Eleonora to pure olivine powders and to a typical Atype asteroid is shown in Fig. 19. Although the band depth for (354) Eleonora is $~ 2.5$ deeper than Sasteroids of comparable size, it is much weaker than either pure olivine powders or a typical A-type asteroid. Moreover, the spectral slope across the $1 \mu \mathrm{m}$ feature is substantially lower for Eleonora $(\Delta \operatorname{Ref} / \Delta \lambda=0.046 \mu \mathrm{m}-1)$ than for the olivine powders $(0.134$ for the $<45 \mu \mathrm{m}$ sample to 0.269 for $250-500$ $\mu \mathrm{m}$ size sample). It is evident from Fig. 19, that the surface material of (354) Eleonora is neither a clean olivine assemblage nor an A-type object.

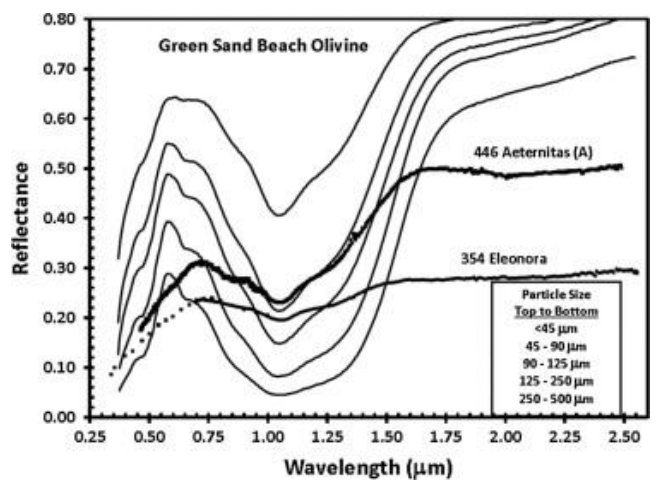

Fig. 19. Spectral albedo curve (reflectance versus wavelength) for (354) Eleonora compared to spectral albedo curves for Green Sand Beach olivine powders and to A-type asteroid (446 Aeternitas). The Aeternitas spectrum is from Sunshine et al. (2007) and the SMASS-I survey. The spectral albedo curves for Eleonora and Aeternitas were derived by multiplying the normalized reflectance curves by the albedos of the asteroids, 0.1948 and 0.2361 , respectively. Albedos from the PDS - Small Bodies Node:

(http://sbn.psi.edu/pds/asteroid/EAR_A_5_DDR_ALBEDOS_V1_1/data/albedos.tab).

What mechanism could be responsible for the low overall reflectance of (354) Eleonora, the moderately weak olivine feature, the narrow $1 \mu \mathrm{m}$ olivine feature and the low slope across the $1 \mu \mathrm{m}$ feature? The common default explanation for almost any mismatch between asteroid spectra and laboratory spectra of meteorite samples has been "space weathering".

Space weathering is the general term for the alteration of the optical surfaces on solid bodies exposed to the space environment. The presence of a micrometeorite dominated space weathering process on the lunar surface is well documented and understood (e.g., Pieters et al., 2000, Taylor et al., 2001, Fischer and Pieters, 1994, Noble et al., 2001, Noble et al., 2005, Noble et al., 2007). However, space weathering actually observed on asteroid surfaces and in meteorite regolith breccias generally does not follow the lunar space weathering model (e.g., Gaffey, 2010, Noble et al., 2007, Pieters et al., 2012). It is an open question to what extent the laser and charged particle irradiation simulations of space weathering, which are intended to simulate lunar-style space weathering (e.g., Hiroi and Sasaki, 1999, Hiroi and Sasaki, 
2001, Moroz et al., 1996, Yamada et al., 1999, Sasaki et al., 2002, Sasaki et al., 2003, Marchi et al., 2005, Loeffler et al., 2008, Loeffler et al., 2009), are actually relevant to most asteroids.

Assuming for the moment that these irradiation experiments are relevant to (354) Eleonora, are the spectral properties of Eleonora consistent with such a lunar-style space weathering process? The spectral effects of irradiation on olivine are dramatic. Sasaki et al., 2002, Sasaki et al., 2003 shows a large decrease $(\sim 80 \%$ to $\sim 25 \%)$ in mid-visible $(\sim 0.6 \mu \mathrm{m})$ reflectance with virtually no decrease at $2.5 \mu \mathrm{m}$, resulting a very steeply reddened spectrum (slope $(\Delta \operatorname{Ref} / \Delta \lambda)$ increasing from $\sim 0.07 \mu \mathrm{m}-1$ for the original olivine to $\sim 0.35 \mu \mathrm{m}-1$ for the most heavily irradiated sample. The intensity of the $1 \mu \mathrm{m}$ olivine feature is reduced from $\sim 30 \%$ to $<10 \%$ by the irradiation. Moroz et al., 1996, Yamada et al., 1999, Hiroi and Sasaki, 2001, Kurahashi et al., 2002, Brunetto et al., 2006, Brunetto et al., 2007, Loeffler et al., 2008, Loeffler et al., 2009 and Fu et al. (2012) report similar results from laser and ion irradiation of olivine.

In contrast to the changes exhibited by these space weathering simulations, the spectral albedo curve of (354) Eleonora is uniformly darkened and less "red" than olivine, its primary mafic silicate component. Thus, the difference between the Eleonora spectrum and that of olivine cannot be the result of this lunar style space weathering process. We can also eliminate the styles of space weathering - the natures of which are poorly constrained - exhibited by 433 Eros (slope and band depth unchanged while albedo decreases) and by 243 Ida (slope increases and band depth decreases while albedo remains constant) (Gaffey, 2010). It seems unlikely that "space weathering" is the answer for (354) Eleonora.

Thus the reduced albedo, narrowed absorption feature, and low spectral slope remain to be explained. The mechanism responsible for those changes could provide insight into the nature or properties of (354) Eleonora beyond the dominant mafic silicate phase olivine. It seems reasonable that these spectral properties are either due to changes in the optical properties (i.e., space weathering) or to the presence of one or more additional mineral components on the surface of Eleonora. As noted above, the lunar-style and ion irradiation space weathering options have been largely eliminated. What additional mineral phase could produce the spectral changes when included with an olivine phase?

It appears that an intimate mixture of fine grained olivine and NiFe metal can match the parameters for (354) Eleonora. Cloutis et al. (2015) carried out a spectral study of an extensive set of olivine-NiFe metal mixtures and quantified the various parameters. Fig. 20 shows the results of that work for the following spectral parameters versus olivine abundance: FWHM, albedo (reflectance at $0.56 \mu \mathrm{m}$ ), spectral slope across the $1 \mu \mathrm{m}$ feature, and depth of the $1 \mu \mathrm{m}$ feature. The corresponding values of these parameters for (354) Eleonora are shown by the dashed horizontal lines. Although the Band I minimum shifts toward shorter wavelength with increasing metal content, the band center remains unchanged until the olivine feature is significantly weakened and band center determinations become less robust. 

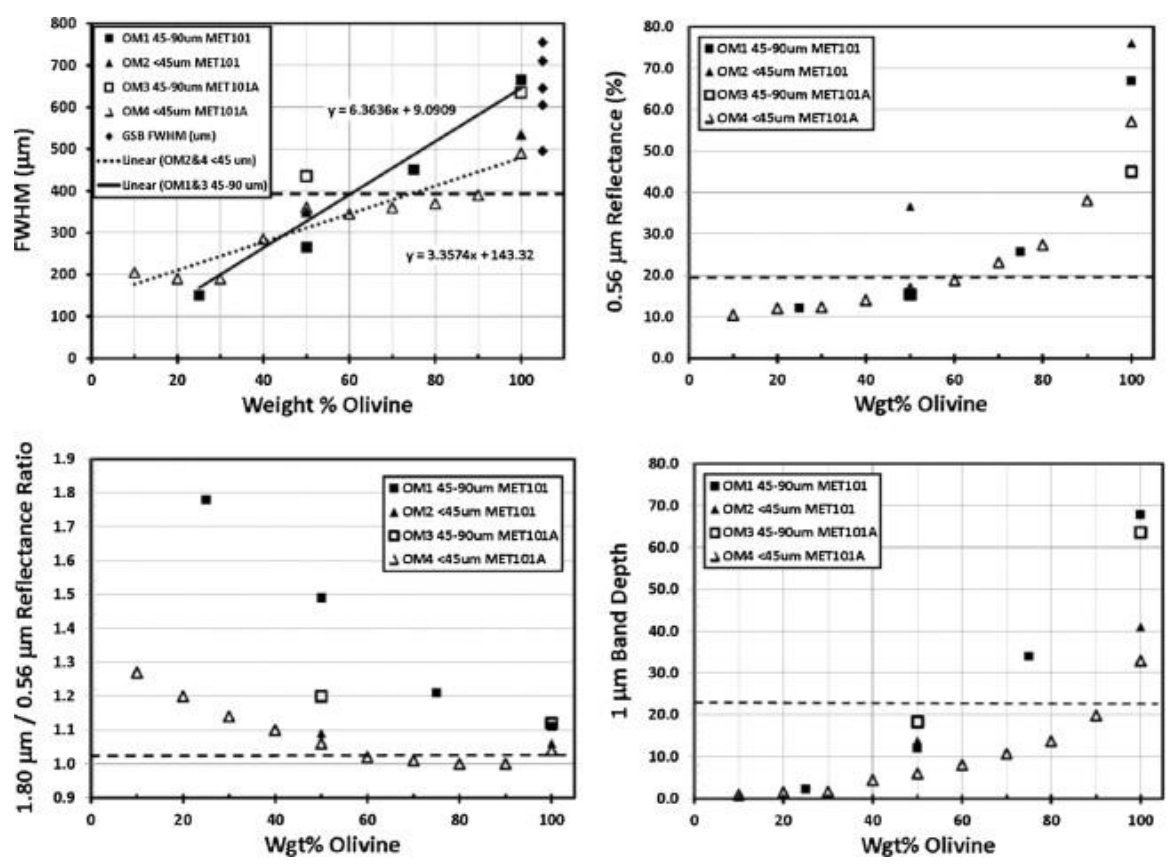

Fig. 20. Upper left: full width at half maximum (FWHM) of the $1 \mu \mathrm{m}$ olivine feature in intimate mixtures of olivine and iron meteorite powders (data from Cloutis et al., 2015). The triangles and squares represent $<45$ and $45-90 \mu \mathrm{m}$ powders, respectively. The composition of the olivine component is Fa9.6. The metal phase (MET101 \& MET101A) is from the Odessa iron meteorite (coarse octahedrite; group IAB; $7.2 \% \mathrm{Ni}$ ). For MET101 the meteorite metal powder, $<45$ and 45 $90 \mu \mathrm{m}$, was beaten in an alumina mortar and pestle to create more equant grains. For MET101A, the iron meteorite powder underwent no further treatment after grinding, sieving, and magnetic separation. The FWHM for Green Sand Beach olivine sample are plotted as solid diamonds at the right for comparison, with the smallest size fraction $(<45 \mu \mathrm{m})$ at the bottom and the coarsest size fraction $(250-500 \mu \mathrm{m})$ at the top. Linear functions have been fit to the $<45$ and $45-90 \mu \mathrm{m}$ mixtures. Upper right: Reflectance at $0.56 \mu \mathrm{m}$ ( albedo). Lower left: Spectral slope across $1 \mu \mathrm{m}$ feature. Lower right: $1 \mu \mathrm{m}$ band depth. Values for each of these parameters for (354) Eleonora are shown by the dashed line.

Although there is some spread between the $<45$ and the $45-90 \mu \mathrm{m}$ size fractions, an olivine abundance of $\sim 60-70 \%$ will reasonably match all four spectral parameters. In particular, the narrow FWHM for Eleonora is matched by such a NiFe metal-olivine mixture while none of the mechanisms discussed previously could plausibly produce such a narrow feature.

\section{Questions}

Based on the preceding discussion, we interpret the surface material of (354) Eleonora to be a relatively uniform intimate mixture of fine-grained olivine (60-70\%) and $\mathrm{NiFe}$ metal. The composition of the olivine is $\sim$ Fo61-71. A weak $2 \mu \mathrm{m}$ absorption feature is consistent with the presence of as much as $\sim 8$ $10 \%$ pyroxene in the silicate assemblage. The composition of the pyroxene phase is weakly constrained at $\sim$ Fs50Wo10.

There are two possible origins for a surface assemblage of intimately mixed fine grained mafic silicates and $\mathrm{NiFe}$ metal. The first possibility is normal impact produced regolith comminution of a coarse grained olivine-metal substrate assemblage analogous to a pallasite. Normal regolith processes can generate finegrained regoliths from brittle substrates. Impacts onto a pallasite-like bedrock (coarse grained olivine in a $\mathrm{NiFe}$ metal matrix) cooled below the brittle-ductile transition temperature for $\mathrm{NiFe}$ metal would generate a regolith. Although initial retention of ejecta fragments would be low, once even a thin particulate layer 
was built up, losses would decrease significantly and the regolith fragments would be recycled, decreasing the mean particle size.

The brittle-ductile transition temperature has been estimated at $\sim 200 \mathrm{~K}$ or lower by a number of investigators from experiments on iron meteorites. The Henbury, Gibeon, El Sampal and Arispe octahedrites ( 7-8.5\% Ni) are brittle at $\sim 200 \mathrm{~K}$ (e.g., Remo and Johnson, 1974, Remo and Johnson, 1975, Matsui and Schultz, 1984). The transition temperature is sensitive to the nickel content, higher nickel alloys have decreasing transition temperatures. High nickel-iron alloys remains ductile down to 0 K. Consistent with this, Johnson et al. (1979) noted that the nickel-rich ataxite Hoba (17\% Ni) was ductile at $195 \mathrm{~K}$ and brittle at $77 \mathrm{~K}$, with a brittle-ductile transition below 140-150 K. Using the Burbine et al. (2009) formula for Eleonora gives a surface temperature of $172 \mathrm{~K}$ at perihelion (2.473 AU) and $153 \mathrm{~K}$ at aphelion (3.119 AU). If the bedrock of (354) Eleonora consisted of olivine in a low nickel alloy, the substrate should be brittle most of the time.

Alternately, the bedrock itself could consist of a fine-grained intimate mixture of $\mathrm{NiFe}$ metal and olivine. If a chondritic precursor is heated, two eutectic melts appear at $\sim 950{ }^{\circ} \mathrm{C}$, a basaltic melt and a $\mathrm{NiFe}-\mathrm{FeS}$ melt. (Eutectic basaltic melting in a dry chondritic assemblage occurs at $\sim 1020{ }^{\circ} \mathrm{C}$.) The interfacial energy of the melt relative to the sold matrix determines the mobility of the melt. Melts with low interfacial energies (dihedral angle $<60^{\circ}$ ) can easily diffuse along cracks and grain boundaries while those with high interfacial energies (dihedral angle $>60^{\circ}$ ) remain trapped in the solid matrix until high degrees of melting are attained (e.g. Minarik et al., 1996, Minarik, 2003, McCoy et al., 2006). The interfacial energy of the basaltic melt in a mafic silicate assemblage is very low so that the melt can easily percolate through small cracks and along grain boundaries and escape from the melting zone. By contrast the interfacial energy of the NiFe-FeS melt in the mafic silicate matrix is very high so that the metallic melt remains trapped in the matrix until the melt fraction approaches 50\% (e.g., Taylor, 1992).

So if the basaltic melt is continuously extracted from a chondritic precursor during partial melting, the total melt fraction will remain low and the $\mathrm{NiFe}-\mathrm{FeS}$ droplets will remain trapped in the matrix. As the basaltic component is removed the residual assemblage becomes progressively enriched in olivine. If heating is terminated before significant melting of the olivine phase occurs, the final residual assemblage will consist of a predominantly olivine matrix with fine-grained $\mathrm{NiFe}-\mathrm{FeS}$ blebs scattered throughout. If such an assemblage is comminuted by regolith processes, it would result in an intimate mixture of finegrained olivine and $\mathrm{NiFe}$ metal similar to that inferred from our analysis.

The residual model outlined above is constrained by igneous processes. During the partial melting, the iron content (Fa) of the residual olivine decreases (e.g., Taylor, 1992, Sunshine et al., 2007). For an Hchondrite precursor, by the temperature at which the basaltic (i.e., pyroxene) component is exhausted, the Fa content of the olivine has dropped from $\sim 20 \%$ to $\sim 5 \%$ (or put another way, the molar Fo content has increased from $80 \%$ to $95 \%$ ).

For the more oxidized LL- and R-chondrites, the initial iron content is higher ( $\sim \mathrm{Fa} 26-32 \& \sim \mathrm{Fa} 37-40)$. In such precursor assemblages, the smaller "basaltic" component and the higher initial fayalite content would result in a higher final fayalite content. Fig. 2 of Sunshine et al. (2007) shows the results of a melting experiment on an R-chondrite. By $\sim 1140{ }^{\circ} \mathrm{C}(\sim 17 \%$ melting) the silicate residue is $\sim 100 \%$ olivine with a composition of $\sim$ Fs38 ( Fo62). Melting to about $40 \%$ would lower the olivine to $\sim$ Fa30 ( $\sim$ Fo70). However, the higher fayalite (and ferrosilite) in these assemblages is at the expense of the initial $\mathrm{NiFe}$ abundance ( $\sim 4 \%$ and $<1 \%$, respectively). These assemblages do contain $\mathrm{FeS}$ and $\mathrm{NiFeS}(\sim 6 \%$ and up to $\sim 8 \%$, respectively) which would form a high interfacial energy melt (dihedral angle $\sim 90^{\circ}-$ Minarik et al., 1996). 
Unless we invoke a starting material significantly richer in $\mathrm{NiFe}$ metal and total iron than chondrites, it would be difficult to produce an assemblage with $\sim$ Fa30 and an abundant $(\sim 30-40 \%)$ NiFe metal phase by this partial melting mechanism.

Could the substrate for (354) Eleonora actually be a pallasite assemblage? Based on our derived olivine composition of $\sim$ Fo61-71, the main group pallasites with olivine compositions near Fo88 would appeared to be ruled out. However, the olivine in the Eagle Station group pallasites ( Fo80) falls near the uncertainty limit of our derived composition. This should not be construed to be a genetic link between the Eagle Station group pallasites and Asteroid (354) Eleonora, but rather the suggestion of generally similar lithologies.

Sunshine et al. (2007) used the MGMFIT routine to fit the spectrum of (354) Eleonora combined from the SMASS-II and MIT NEOSR spectra (see Fig. 12). Their analysis derived an olivine composition of Fa10 (Fo90). The use of the SMASS-II spectrum produces a significantly different spectral curve at the short wavelength edge of the olivine feature than if the SMASS-I spectrum had been used. It is not clear whether and to what degree, substituting the SMASS-I or S3OS2 spectra would change their results. At this point all we can do is note the discrepancy with our result of $\sim$ Fa34 $(\sim$ Fo66).

A third possibility, suggested by Fig. 4, is that (354) Eleonora is a fine grained CK6-type assemblage. Fig. 21 plots the reflectance spectra of (354) Eleonora and the A-type Asteroid (446) Aeternitas versus the $<63$ micrometer sample of the CK6 chondrite LEW 87009. Although the band intensity, spectral curve longwards of $\sim 0.70 \mu \mathrm{m}$, and the reflectance (albedo) of the CK6 sample are comparable to those of (354) Eleonora, there is a significant difference shortwards of $\sim 0.70 \mu \mathrm{m}$. The Eleonora spectrum drops off steeply shortwards of $\sim 0.70 \mu \mathrm{m}$, while the CK6 spectrum does not roll over until $\sim 0.55 \mu \mathrm{m}$. Cloutis et al. (2012b) explored the spectral systematics of CK chondrites, all of which show this similar short wavelength roll over. While this possibility cannot be ruled out entirely, this spectral mismatch would mitigate against a CK6 interpretation for (354) Eleonora.

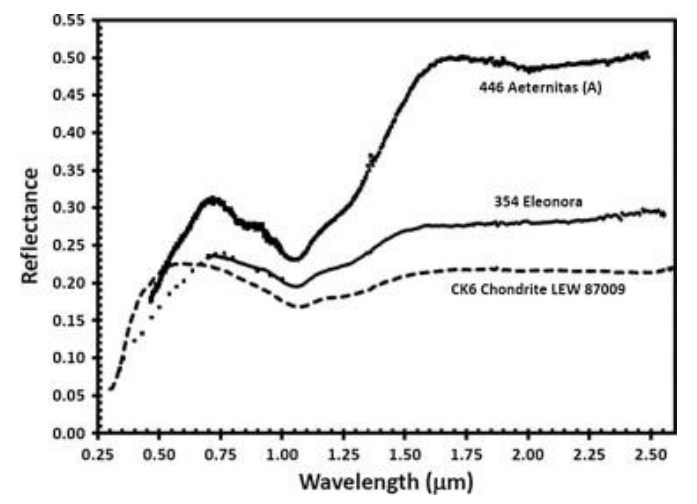

Fig. 21. Spectral albedo curve of (354) Eleonora compared to those for (446) Aeternitas (Sunshine et al., 2007) and the CK6 chondrite LEW 87009 (Cloutis et al., 2012b; RELAB spectral database sample MP-LAM-005).

\section{Summary and conclusions}

The possibility that the differences between the visible and very near infrared spectra of (354) Eleonora from the three major asteroid CCD surveys (SMASS-I, SMASS-II, S3OS2) could arise from lithological heterogeneities on the surface of Eleonora can be excluded. By contrast the near-infrared spectra of (354) Eleonora show only minor differences, primarily in the overall spectral slope, most of which can be attributed to slight differences in the standard stars used to calibrate the data. 
In the case of (354) Eleonora, three very different compositional interpretations would result from the three CCD spectral data sets. The inconsistencies remain to be explained. It will be important to determine whether this discrepancy is a unique case or whether it is part of a larger pattern of inconsistencies. If it is a pervasive problem, it calls into question interpretations based on these CCD data.

Spectral parameters were extracted from the 226 NIR spectral of (354) Eleonora obtained in June 2011 using the SpeX instrument on the NASA Infrared Telescope Facility at Mauna Kea Observatory. These parameters are most consistent with a surface assemblage of fine-grained intimately mixed olivine $(\sim 60$ $70 \%, \sim$ Fo61-71) and low nickel $(<\sim 7 \% \mathrm{Ni}) \mathrm{NiFe}$ metal. The surface assemblage appears to contain a small component $(\sim 8-10 \%)$ of igneous pyroxene (weakly constrained at $\sim$ Fs50Wo10). The parent lithology of the surface regolith may be similar to a pallasite assemblage, although none of the three known types of pallasites are good matches.

\section{Acknowledgments}

This research has been supported by NASA Planetary Geology \& Geophysics Program grants NNX10AG45G and NNX11AN84G and Near-Earth Object/Planetary Astronomy Program grant NNX12AG12G. Part of the data utilized in this publication were obtained and made available by the MIT-UH-IRTF Joint Campaign for NEO Reconnaissance. The IRTF is operated by the University of Hawaii under Cooperative Agreement no. NCC 5-538 with the National Aeronautics and Space Administration, Office of Space Science, Planetary Astronomy Program. The MIT component of this work is supported by NASA grant 09-NEOO009-0001, and previously by the National Science Foundation under Grant No. 0506716. We also are grateful for the comments from Juan Sanchez and an anonymous reviewer which improved the manuscript.

\section{References}

Adams, J.B., 1974. Visible and near-infrared diffuse reflectance spectra of pyroxenesas applied to remote sensing of solid objects in the Solar System. J. Geophys. Res. 79, 4829-4836.

Bell, J.F., Owensby, P.D., Hawke, B.R., Gaffey, M.J., 1988. The 52-color asteroid survey: Final results and interpretation. Proc. Lunar Sci. Conf. XIX, 57-58(Abstract) (EAR-A-RDR-3-52COLORV2.1. NASA Planetary Data System, 1995).

Berlin, J., 2003. Mineralogisch-petrographische Untersuchungen an derchondritischen Breccie Rumuruti. Doctoral Thesis, Fachbereich Geowissenschaften der Freien Universität, Berlin.

Berlin, J., Lingemann, C.M., Stöffler, D., 2003. Visible and near-infrared reflectance spectra of Rumuruti. Lunar Planet. Sci. XXXIV. Abstract 1764.

Binzel, R.P., Birlan, M., Bus, S.J., Harris, A.W., Rivkin, A.S., Fornasier, S., 2004. Spectral observations for near-Earth objects including potential target 4660 Nereus: Results from Meudon remote observations at the NASA Infrared Telescope Facility (IRTF). Planet. Space Sci. 52, 291-296.

Brunetto, R., Romano, F., Blanco, A., Fonti, S., Martino, M., Orofino, V., Verrienti, C.,2006. Space weathering of silicates simulated by nanosecond pulse UV excimer laser. Icarus 180, 546-554.

Brunetto, R., Roush, T.L., Marra, A.C., Orofino, V., 2007. Optical characterization of laser ablated silicates. Icarus 191, 381-393.

Burbine, T.H., Binzel, R.P., 2002. Small main-belt asteroid spectroscopic survey in the infrared. Icarus $159,468-499$. 
Burbine, T.H., McCoy, T.J., Hinrichs, J.L., Lucey, P.G., 2006. Spectral properties of angrites. Meteoritics and Planetary Science 41, 1139-1145.

Burbine, T.H., Buchanan, P.C., Dolkar, T., Binzel, R.P., 2009. Pyroxene mineralogies of near-Earth vestoids. Meteorit. Planet. Sci. 44, 1331-1341.

Burchi, R., D’Ambrosio, V., Tempesti, P., Lanciano, N., 1985. Rotational properties of Asteroids 2, 12, 80, 145 and 354 obtained by photoelectric photometry. Astron. Astrophys. Suppl. Ser. 60, 9-15.

Bus, S.J., Binzel, R.P., 2002a. Phase II of the small main-belt asteroid spectroscopic survey - The observations. Icarus 158, 106-145.

Bus, S.J., Binzel, R.P., 2002b. Phase II of the small main-belt asteroid spectroscopic survey - A feature based taxonomy. Icarus 158, 146-177.

Chang, Y.C., Chang, C., 1963. Photometric investigations of seven variable asteroids, II. Acta Astron. Sin. 11, 139-149.

Chapman, C.R., Gaffey, M.J., 1979. TRIAD: IV. Spectral reflectances. In: Gehrels, T.,Matthews, M.S. (Eds.), Asteroids. University of Arizona Press, Tucson, pp. 1064-1089 (PDS Small Bodies Node).

Chapman, C.R., McCord, T.B., Johnson, T.V., 1973. Asteroid spectral reflectivities. Astron. J. 78, 126140.

Cloutis, E.A., Gaffey, M.J., 1991. Pyroxene spectroscopy revisited: Spectral-compositional correlations and relationship to geothermometry. J. Geophys. Res.: Planets 96, 22809-22826.

Cloutis, E.A., Gaffey, M.J., Jackowski, T.L., Reed, K.L., 1986. Calibration of phase abundance, composition, and particle size distribution for olivine-orthopyroxene mixtures from reflectance spectra. J. Geophys. Res. 91, 11641-11653.

Cloutis, E.A. et al., 2010. Spectral reflectance properties of ureilites. Meteorit. Planet. Sci. 45, 16681694.

Cloutis, E.A., Hudon, P., Hiroi, T., Gaffey, M.J., Mann, P., 2012a. Spectral reflectance properties of carbonaceous chondrites: 8 . 'Other' carbonaceous chondrites: $\mathrm{CH}$, ungrouped, polymict, xenolithic inclusions, and R chondrites. Icarus 221, 984-1001.

Cloutis, E.A., Hudon, P., Hiroi, T., Gaffey, M.J., 2012b. Spectral reflectance properties of carbonaceous chondrites: 7. CK chondrites. Icarus 221, 911-924.

Cloutis, E.A., Sanchez, J.A., Reddy, V., Gaffey, M.J., Binzel, R.P., Burbine, T.H., Hardersen, P.S., Hiroi, T., Lucey, P.G., Sunshine, J.M., Tait, K.T., 2015. Olivine-metal mixtures: Spectral reflectance properties and application to asteroid reflectance spectra. Icarus, in press (Available online).DM02. <http://smass.mit.edu/minus.html\#unpublished>.

Drummond, J.D., Weidenschilling, S.J., Chapman, C.R., Davis, D.R., 1991. Photometric Geodesy of main-belt asteroids: IV. An updated analysis of light curves for poles, periods, and shapes. Icarus $89,44-64$.

Dunn, T.L., McCoy, T.J., Sunshine, J.M., McSween Jr., H.Y., 2010. A coordinated spectral, mineralogical, and compositional study of ordinary chondrites. Icarus208, 789-797. 
Fischer, E.M., Pieters, C.M., 1994. Remote determination of exposure degree and iron concentration of lunar soils using VIS-NIR spectroscopic methods. Icarus 111,475-488.

Fu, X., Zou, Y., Zheng, Y., Ouyang, Z., 2012. Effects of space weathering on diagnostic spectral features: Results from He+irradiation experiments. Icarus 219, 630-640.

Gaffey, M.J., 2010. Space weathering and the interpretation of asteroid reflectance spectra. Icarus 209, $564-574$.

Gaffey, M.J., 1997. Surface lithologic heterogeneity of asteroid 4 Vesta. Icarus 127,130-157.

Gaffey, M.J., Bell, J.F., Brown, R.H., Burbine, T.H., Piatek, J., Reed, K.L., Chaky, D.A.,1993. Mineralogical variations within the S-type asteroid class. Icarus 106, 573-602.

Gaffey, M.J., Cloutis, E.A., Kelley, M.S., Reed, K.L., 2002. Mineralogy of asteroids. In: Bottke, W.F., Cellino, A., Paolicchi, P., Binzel, R.P. (Eds.), Asteroids III. Univ. of Arizona Press, pp. 183-204.

Gietzen, K.M., Lacy, C.H.S., Ostrowski, D.R., Sears, D.W.G., 2012. IRTF observations of S complex and other asteroids: Implications for surface compositions, the presence of clinopyroxenes, and their relationship to meteorites. Meteorit. Planet. Sci. 47, 1789-1808.

Goodrich, C.A., Van Orman, J.A., Wilson, L., 2007. Fractional melting and smelting on the ureilite parent body. Geochim. Cosmochim. Acta 71, 2876-2895.

Gradie, J., Veverka, J., 1981. Effects of body shape on disk-integrated spectral reflectance. Proc. Lunar Sci. Conf. 12, 1769-1779.

Gradie, J., Veverka, J., 1982. When are spectral reflectance curves comparable?Icarus 49, 109-119.

Gradie, J., Veverka, J., 1986. The wavelength dependence of phase coefficients. Icarus66, 455-467.

Groeneveld, I., Kuiper, G.P., 1954. Photometric studies of asteroids. I. Astrophys. J.120, 200-220.

Hardorp, J., 1978. The Sun among the stars. I. A search for solar spectral analogs. Astron. Astrophys. 63, 383-390.

Hardorp, J., 1980. The Sun among the stars. II - Solar color, Hyades metal content, and distance. Astron. Astrophys. 88, 334-344.

Hinrichs, J.L., Lucey, P.G., 2002. Temperature-dependent near-infrared spectral properties of minerals, meteorites, and lunar soil. Icarus 155, 169-180.

Hiroi, T., Sasaki, S., 1999. Space weathering of olivine as a key to understanding S/A/R/V asteroids and lunar soils. Antarct. Meteorit. Res. 14, 34-36, Nat. Inst. Polar Res., Tokyo. Hiroi, T., Sasaki, S., 2001. Importance of space weathering simulation products in compositional modeling of asteroids: 349 Dembowska and 446 Aeternitas as examples. Meteorit. Planet. Sci. 36, 1587-1596.

Howell, E.S., Merenyi, E., Lebofsky, L.A., 1994. Classification of asteroid spectra usinga neural network. J. Geophys. Res. 99, 10847-10865.

Johnson, A.A., Remo, J.L., Davis, R.B., 1979. The low temperature impact properties ofthe meteorite Hoba. J. Geophys. Res. 84, 1683-1688. 
Kaasalainen, M., Torppa, J., Piironen, J., 2002. Models of twenty asteroids from photometric data. Icarus 159, 369-395.

Kallemeyn, G.W., Rubin, A.E., Wasson, J.T., 1996. The compositional classification of chondrites: VII. The R chondrite group. Geochim. Cosmochim. Acta 60, 2243-2256.

King, T.V.V., Ridley, W.I., 1987. Relation of the spectroscopic reflectance of olivine to mineral chemistry and some remote sensing implications. J. Geophys. Res. 92,11457-11469.

Kurahashi, E., Yamanaka, C., Nakamura, K., Sasaki, S., 2002. Laboratory simulation of space weathering: ESR measurements of nanophase metallic iron in laser-irradiated materials. Earth Planets Space 54, e5-e7.

Lagerkvist, C.-I., Magnusson, P. (Eds.), 2011. Asteroid Photometric Catalog V1.1.EAR-A-3-DDR-APCLIGHTCURVE-V1.1. NASA Planetary Data System.

Lazzaro, D., Angeli, C.A., Carvano, J.M., Mothé-Diniz, T., Duffard, R., Florczak, M., 2004.S3OS2: The visible spectroscopic survey of 820 asteroids. Icarus 172, 179-220.Loeffler, M.J., Baragiola, R.A., Murayama, M., 2008. Laboratory simulations of redeposition of impact ejecta on mineral surfaces. Icarus 196, 285-292.

Loeffler, M.J., Dukes, C.A., Baragiola, R.A., 2009. Irradiation of olivine by $4 \mathrm{keV} \mathrm{He+:Simulation} \mathrm{of}$ space weathering by the solar wind. J. Geophys. Res.: Planets 114(E03003), 2009.http://dx.doi.org/10.1029/2008JE003249.

Lucey, P.G., Keil, K., Whitely, R., 1998. The influence of temperature on the spectra of the A-asteroids and implications for their silicate chemistry. J. Geophys. Res.: Planets 103, 5865-5871.

Magnusson, P., 1990. Spin vectors of 22 large asteroids. Icarus 85, 229-240.

Marchi, S., Brunetto, R., Magrin, S., Lazzarin, M., Gandolfi, D., 2005. Space weathering on near-Earth and main belt silicate-rich asteroids: Observation sand ion irradiation experiments. Astron. Astrophys. 443, 769-775.

Matsui, T., Schultz, P.H., 1984. On the brittle-ductile behavior of iron meteorites: New experimental constraints. J. Geophys. Res. 89 (1), C323-C328 (Proc. Lunar Plant. Sci. Conf. 15th).

McCoy, T.J., Mittlefehldt, D.W., Wilson, L., 2006. Asteroid differentiation. In:McSween, H.Y., Lauretta, D.S., Jr. (Eds.), Meteorites and the Early Solar System II. University of Arizona Press, pp. 733746.

Minarik, B., 2003. The core of planet formation. Nature 422, 126-128.

Minarik, W.G., Ryerson, F.J., Watson, E.B., 1996. Textural entrapment of core-forming melts. Science 272, 530-533.

Minton, D.A., Malhotra, R., 2010. Dynamical erosion of the asteroid belt and implications for large impacts in the inner Solar System. Icarus 207, 744-757.

Moroz, L.V., Fisenko, A.V., Semjonova, L.F., Pieters, C.M., Korotaeva, N.N., 1996.Optical effects of regolith processes on $\mathrm{S}$-asteroids as simulated by laser shots on ordinary chondrite and other mafic materials. Icarus 122, 366-382. 
Moroz, L., Schade, U., Wäsch, R., 2000. Reflectance spectra of olivine-orthopyroxene bearing assemblages at decreased temperatures: Implications for remote sensing of asteroids. Icarus 147, 79-93.

Noble, S.K. et al., 2001. The optical properties of the finest fraction of lunar soil: Implications for space weathering. Meteorit. Planet. Sci. 36, 31-42.

Noble, S.K., Keller, L.P., Pieters, C.M., 2005. Evidence of space weathering in regolithbreccias I: Lunar regolith breccias. Meteorit. Planet. Sci. 40, 397-408.

Noble, S.K., Pieters, C.M., Keller, L.P., 2007. An experimental approach to understanding the optical effects of space weathering. Icarus 192, 629-642.

Pieters, C.M. et al., 2000. Space weathering on airless bodies: Resolving a mystery with lunar samples. Meteorit. Planet. Sci. 35, 1101-1107.

Pieters, C.M. et al., 2012. Distinctive space weathering on Vesta from regolith mixing processes. Nature 491, 79-86.

Piironen, J.O., Poutanen, M., Di Martino, M., Zappalà, V., 1985. UB V observations and pole determinations of Asteroids 15 Eunomia and 354 Eleonora. Astron. Astrophys. Suppl. Ser. 61, 299-302.

Rayner, J.T. et al., 2003. SpeX: A medium-resolution 0.8-5.5 micron spectrograph and imager for the NASA Infrared Telescope Facility. Publ. Astron. Soc. Pacific115, 362-382.

Reddy, V. et al., 2011a. Photometric, spectral phase and temperature effects on Vesta and HED meteorites: Implications for Dawn mission. Icarus 217, 153-168.

Reddy, V., Nathues, A., Gaffey, M.J., Schaeff, S., 2011b. Mineralogical characterization of potential targets for the ASTEX mission scenario. Planet. Space Sci. 59, 772-778.

Reddy, V., Sanchez, J.A., Nathues, A., Moskovitz, N.A., Li, J.-Y., Cloutis, E.A., Archer, K.,Tucker, R.A., Gaffey, M.J., Mann, J.P., Sierks, H., Schade, U., 2012. Photometric, spectral phase and temperature effects on 4 Vesta and HED meteorites: Implications for the Dawn mission. Icarus $217,153-168$.

Remo, J.L., Johnson, A.A., 1974. The ductile-brittle transition in meteoritic irons. Meteoritics 9, 209213.

Remo, J.L., Johnson, A.A., 1975. A preliminary study of the ductile-brittle transition under impact conditions in material from an octahedrite. J. Geophys. Res. 80,3744-3748.

Roush, T.L., 1984. Effects of Temperature on Remotely Sensed Mafic Mineral Absorption Features. MS Thesis, Univ. of Hawaii, Honolulu. Roush, T.L., Singer, R.B., 1986. Gaussian analysis of temperature effects on the reflectance spectra of mafic minerals in the 1-lm region. J. Geophys. Res. 91,10301-10308.

Sanchez, J.A., Reddy, V., Nathues, A., Cloutis, E.A., Mann, P., Hiesinger, H., 2012.Phase reddening on near-Earth asteroids: Implications for mineralogical analysis, space weathering and taxonomic classification. Icarus 220, 36-50. 
Sanchez, J.A., Reddy, V., Kelley, M.S., Cloutis, E.A., Bottke, W.F., Nesvorny', D., Lucas, M.P., Hardersen, P.S., Gaffey, M.J., Abell, P.A., Le Corre, L., 2014. Olivine-dominated asteroids: Mineralogy and origin. Icarus 228, 288-300.

Sasaki, S., Hiroi, T., Nakamura, K., Hamabe, Y., Kurahashi, E., Yamada, M., 2002.Simulation of space weathering by nanosecond pulse laser heating: Dependence on mineral composition, weathering trend of asteroids and discovery of nanophase iron particles. Adv. Space Res. 29, 783-788.

Sasaki, S., Kurahashi, E., Yamanaka, C., Nakamura, K., 2003. Laboratory simulation of space weathering: Changes of optical properties and TEM/ESR confirmation of nanophase metallic iron. Adv. Space Res. 31, 2537-2542.

Singer, R.B., Roush, T.L., 1985. Effects of temperature on remotely sensed mineral absorption features. J. Geophys. Res. 90, 12434-12444.

Sunshine, J.M., Pieters, C.M., 1998. Determining the composition of olivine from reflectance spectra. J. Geophys. Res. 103, 13675-13688.

Sunshine, J.M., Bus, S.J., Corrigan, C.M., McCoy, T.J., Burbine, T.H., 2007. Olivine-dominated asteroids and meteorites: Distinguishing nebular and igneoushi stories. Meteorit. Planet. Sci. 42, 155-170.

Taylor, G.J., 1992. Core formation in asteroids. J. Geophys. Res.: Planets 97, 14717-14726.Taylor, L.A. et al., 2001. The effects of space weathering on Apollo 17 mare soils: Petrographic and chemical characterization. Meteorit. Planet. Sci. 36, 285-300.

Tedesco, E.F., Tholen, D.J., Zellner, B., 1982. The eight-color asteroid survey: Standard stars. Astron. J. $87,1585-1592$.

Tedesco, E.F., Williams, J.G., Matson, D.L., Veeder, G.J., Gradie, J.C., Lebofsky, L.A.,1989. A threeparameter asteroid taxonomy. Astron. J. 97, 580-606.

Tholen, D.J., 1984. Asteroid Taxonomy from Cluster Analysis of Photometry. PhD Dissertation, University of Arizona, Tucson. 150pp.

Tholen, D.J., Barucci, M.A., 1989. Asteroid taxonomy. In: Binzel, R.P., Gehrels, T.,Matthews, M.S. (Eds.), Asteroids II. University of Arizona Press, Tucson, pp. 298-315.

Xu, S., Binzel, R.P., Burbine, T.H., Bus, S.J., 1995. Small main-belt asteroids pectroscopic survey: Initial results. Icarus $115,1-35$.

Yamada, M. et al., 1999. Simulation of space weathering of planet forming materials: Nanosecond pulse laser irradiation and proton implantation on olivine and pyroxene samples. Earth Planets Space $51,1255-1265$.

Zappalà, V., van Houten-Groeneveld, I., van Houten, C.J., 1979. Rotation period and phase curve of the Asteroids 349 Dembowska and 354 Eleonora. Astron. Astrophys. Suppl. Ser. 35, 312-321.

Zellner, B., Tholen, D.J., Tedesco, E.F., 1985. The eight-color asteroid survey: Results for 589 minor planets. Icarus $61,355-416$. 